Prepared in cooperation with the Washington State Department of Ecology

\title{
Assessment of Existing Groundwater Quality Data in the Green-Duwamish Watershed, Washington
}

Open-File Report 2019-1131

U.S. Department of the Interior U.S. Geological Survey 
Cover: Duwamish River looking upstream at the South 102nd Street Bridge near Tukwila, Washington. (Photograph by Kathleen E. Conn, U.S. Geological Survey hydrologist.) 


\section{Assessment of Existing Groundwater Quality Data in the Green-Duwamish Watershed, Washington}

By Craig A. Senter, Kathleen E. Conn, Robert W. Black, Wendy B. Welch, and Elisabeth T. Fasser

Prepared in cooperation with the Washington State Department of Ecology

Open-File Report 2019-1131

U.S. Department of the Interior

U.S. Geological Survey 


\section{U.S. Department of the Interior \\ DAVID BERNHARDT, Secretary}

\section{U.S. Geological Survey \\ James F. Reilly II, Director}

U.S. Geological Survey, Reston, Virginia: 2020

For more information on the USGS-the Federal source for science about the Earth,

its natural and living resources, natural hazards, and the environment-visit

https://www.usgs.gov/ or call 1-888-ASK-USGS (1-888-275-8747).

For an overview of USGS information products, including maps, imagery, and publications, visit https:/store.usgs.gov.

Any use of trade, firm, or product names is for descriptive purposes only and does not imply endorsement by the U.S. Government.

Although this information product, for the most part, is in the public domain, it also may contain copyrighted materials as noted in the text. Permission to reproduce copyrighted items must be secured from the copyright owner.

Suggested citation:

Senter, C.A., Conn, K.E., Black, R.W., Welch, W.B., and Fasser, E.T., 2020, Assessment of existing groundwater quality data in the Green-Duwamish watershed, Washington: U.S. Geological Survey Open-File Report 2019-1131, 35 p., https://doi.org/10.3133/ofr20191131.

ISSN 2331-1258 (online) 


\section{Contents}

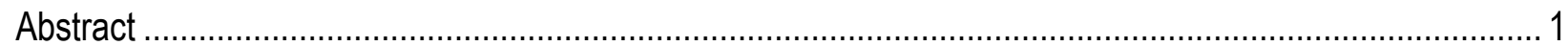

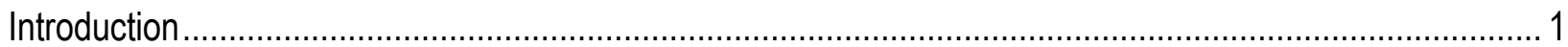

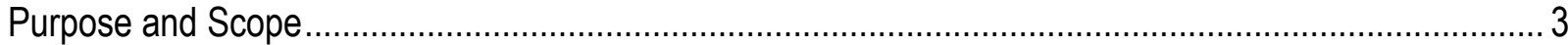

Dataset Compilation and Analysis Methods.................................................................................... 4

Groundwater Quality in the Green-Duwamish Watershed ................................................................ 5

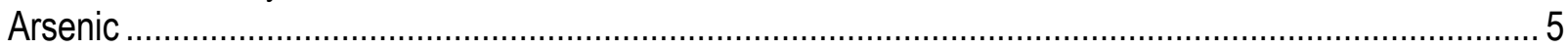

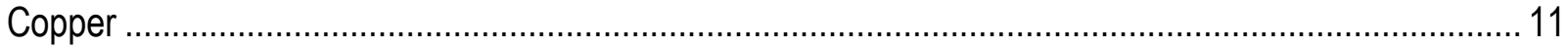

Carcinogenic Polycyclic Aromatic Hydrocarbons........................................................................ 15

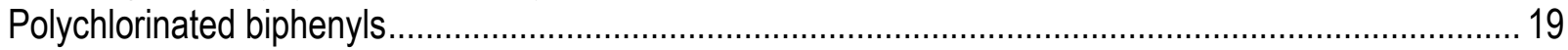

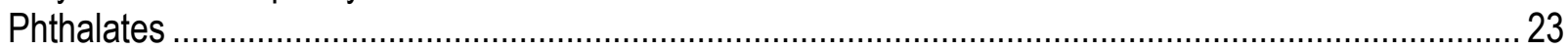

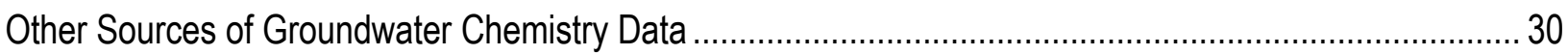

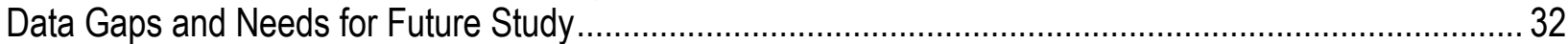

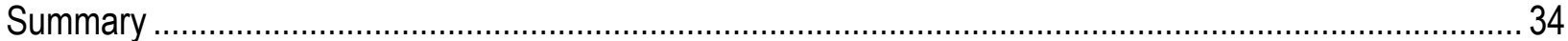

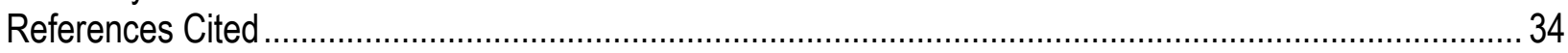

\section{Figures}

Figure 1. Map showing location of the Green-Duwamish River Watershed and subwatersheds, Washington....... 2

Figure 2. Map showing distribution of groundwater sites with arsenic data, Green-Duwamish watershed,

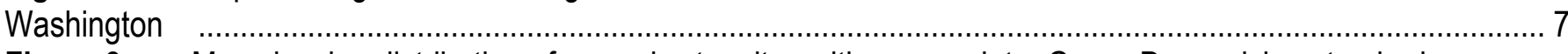

Figure 3. Map showing distribution of groundwater sites with copper data, Green-Duwamish watershed,

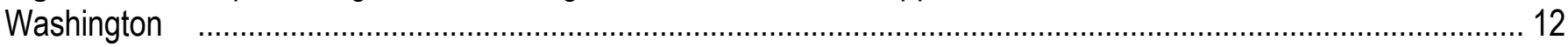

Figure 4. Map showing distribution of groundwater sites with $\mathrm{CPAH}$ data, Green-Duwamish watershed. ............ 16

Figure 5. Map showing distribution of groundwater sites with PCB data, Green-Duwamish watershed,

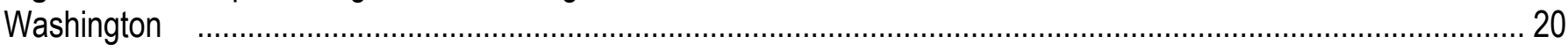

Figure 6. Map showing distribution of groundwater sites with phthalate data, Green-Duwamish watershed,

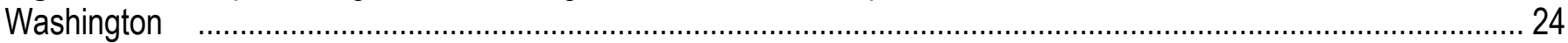

Figure 7. Map showing distribution of groundwater sites with zinc data, Green-Duwamish watershed,

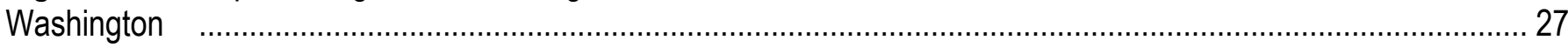

Figure 8. Map showing distribution of groundwater data from designated cleanup sites, Green-Duwamish

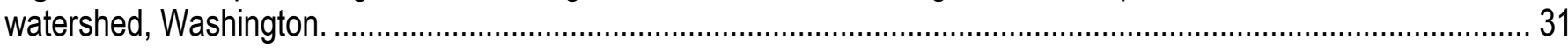

\section{Tables}

Table 1. Summary of data for arsenic in groundwater samples in the Green-Duwamish watershed, Washington

Table 2. Summary of data for copper in groundwater samples in the Green-Duwamish watershed, Washington.

Table 3. Summary of data for carcinogenic polycyclic aromatic hydrocarbons in toxic equivalents in groundwater samples in the Green-Duwamish watershed, Washington.

Table 4. Summary of data for polychlorinated biphenyls in groundwater in the Green-Duwamish watershed, Washington. 21

Table 5. Summary of data for phthalates, represented by the indicator compound bis(2-ethylhexyl)phthalate, in groundwater in the Green-Duwamish watershed, Washington........................................................................ 25

Table 6. Summary of data for zinc in groundwater samples in the Green-Duwamish watershed, Washington. .. 28 


\section{Conversion Factors}

U.S customary units to International System of Units

\begin{tabular}{|c|c|c|}
\hline Multiply & By & To obtain \\
\hline \multicolumn{3}{|c|}{ Length } \\
\hline inch (in.) & 2.54 & centimeter $(\mathrm{cm})$ \\
\hline foot $(\mathrm{ft})$ & 0.3048 & meter $(\mathrm{m})$ \\
\hline \multicolumn{3}{|c|}{ Area } \\
\hline acre & 0.004047 & square kilometer $\left(\mathrm{km}^{2}\right)$ \\
\hline
\end{tabular}

Temperature in degrees Celsius $\left({ }^{\circ} \mathrm{C}\right)$ may be converted to degrees Fahrenheit $\left({ }^{\circ} \mathrm{F}\right)$ as follows:

$$
{ }^{\circ} \mathrm{F}=\left(1.8 \times^{\circ} \mathrm{C}\right)+32
$$

Vertical coordinate information is referenced to the North American Vertical Datum of 1988 (NAVD 88).

Horizontal coordinate information is referenced to the North American Datum of 1983 (NAD 83).

Altitude, as used in this report, refers to distance above the vertical datum.

\section{Supplemental Information}

Concentrations of chemical constituents in water are given either in micrograms per liter $(\mu \mathrm{g} / \mathrm{L})$, micrograms toxic equivalent per liter $(\mu \mathrm{g}$ $T E Q / L)$, picograms per liter, (pg/L) or picograms toxic equivalent per liter (pg TEQ/L).

$\begin{array}{ll}\text { Abbreviations } & \\ \text { BEHP } & \text { bis(2-ethylhexyl)phthalate } \\ \text { cPAH } & \text { carcinogenic polycyclic aromatic hydrocarbon } \\ \text { Ecology } & \text { Washington State Department of Ecology } \\ \text { EIM } & \text { Environmental Information Management System } \\ \text { EPA } & \text { U.S. Environmental Protection Agency } \\ \text { LDW } & \text { Lower Duwamish Waterway } \\ \text { MLE } & \text { maximum likelihood estimation } \\ \text { NWIS } & \text { National Water Information System } \\ \text { PCB } & \text { polychlorinated biphenyl } \\ \text { PLA } & \text { Pollutant Loading Assessment } \\ \text { RKM } & \text { river kilometer } \\ \text { TEQ } & \text { toxic equivalent } \\ \text { USGS } & \text { U.S. Geological Survey }\end{array}$




\title{
Assessment of Existing Groundwater Quality Data in the Green-Duwamish Watershed, Washington
}

\author{
By Craig A. Senter, Kathleen E. Conn, Robert W. Black, Wendy B. Welch, and Elisabeth T. Fasser
}

\begin{abstract}
The United States Geological Survey (USGS) provided technical support to the Washington Department of Ecology (Ecology) in their assessment of the role groundwater plays in contributing pollutant loading to the Green-Duwamish River near Seattle, Washington. Ecology is developing watershed hydrology models of the Green-Duwamish watershed, and need to assign realistic contaminant concentrations to the various Hydrologic Response Units represented in their models. The USGS compiled existing groundwater quality data in the Green-Duwamish watershed, and this report summarizes results and interpretation of the dataset, including identifying data gaps and needs for further research and monitoring. The sources of existing data were the USGS's National Water Information System, Ecology's Environmental Information Management System, and a compilation of several studies by Leidos, a scientific research company. The water-quality parameters of interest included polychlorinated biphenyl (PCB) Aroclors and congeners, phthalates, carcinogenic polycyclic aromatic hydrocarbons (cPAHs), arsenic, copper, and zinc. Results were grouped into the four subwatersheds delineated in Ecology's hydrology models: Duwamish, Lower Green, Soos, and Upper Green. Results from the Duwamish subwatershed were further sub-divided by the USGS into the Lower Duwamish, containing land adjacent to the Lower Duwamish Waterway Superfund site, and the Upper Duwamish, containing the remaining area of the Duwamish subwatershed. Groundwater quality data in the Lower Duwamish were treated separately because there is known contamination in this area. The availability of water quality data varied by subwatershed as follows: phthalate data was only available within the Duwamish, PCB data was available within the Duwamish and Lower Green, cPAH data was available within the Duwamish, Lower Green, and Soos, and data for arsenic, copper, and zinc were available within all four subwatersheds. More than 99 percent of the available data was within the Duwamish subwatershed, identifying a need for additional monitoring of groundwater quality in the other subwatersheds.
\end{abstract}

\section{Introduction}

The Green-Duwamish River originates in the Cascade Mountains near Mount Rainier and travels approximately 150 kilometers northwest through an increasingly developed watershed to Elliott Bay, Puget Sound, Washington, near Seattle (fig. 1). The final 8 kilometers of the river, known as the Lower Duwamish Waterway (LDW), are maintained as a navigable waterway and the sediments are contaminated by past and present anthropogenic activities. In 2001-02, the U.S. Environmental Protection Agency (EPA) and the Washington Department of Ecology (Ecology) required remedial investigations and feasibility studies on this section pursuant to the Federal Superfund Law and the Washington Model Toxics Control Act because of concerns about human health risks from exposure to contaminated sediments. A Record of Decision was issued in 2014 (U.S. Environmental Protection 
Agency, 2014) and included using combinations of dredging, capping, natural sedimentation, and enhanced natural recovery to clean up contaminated sediments.

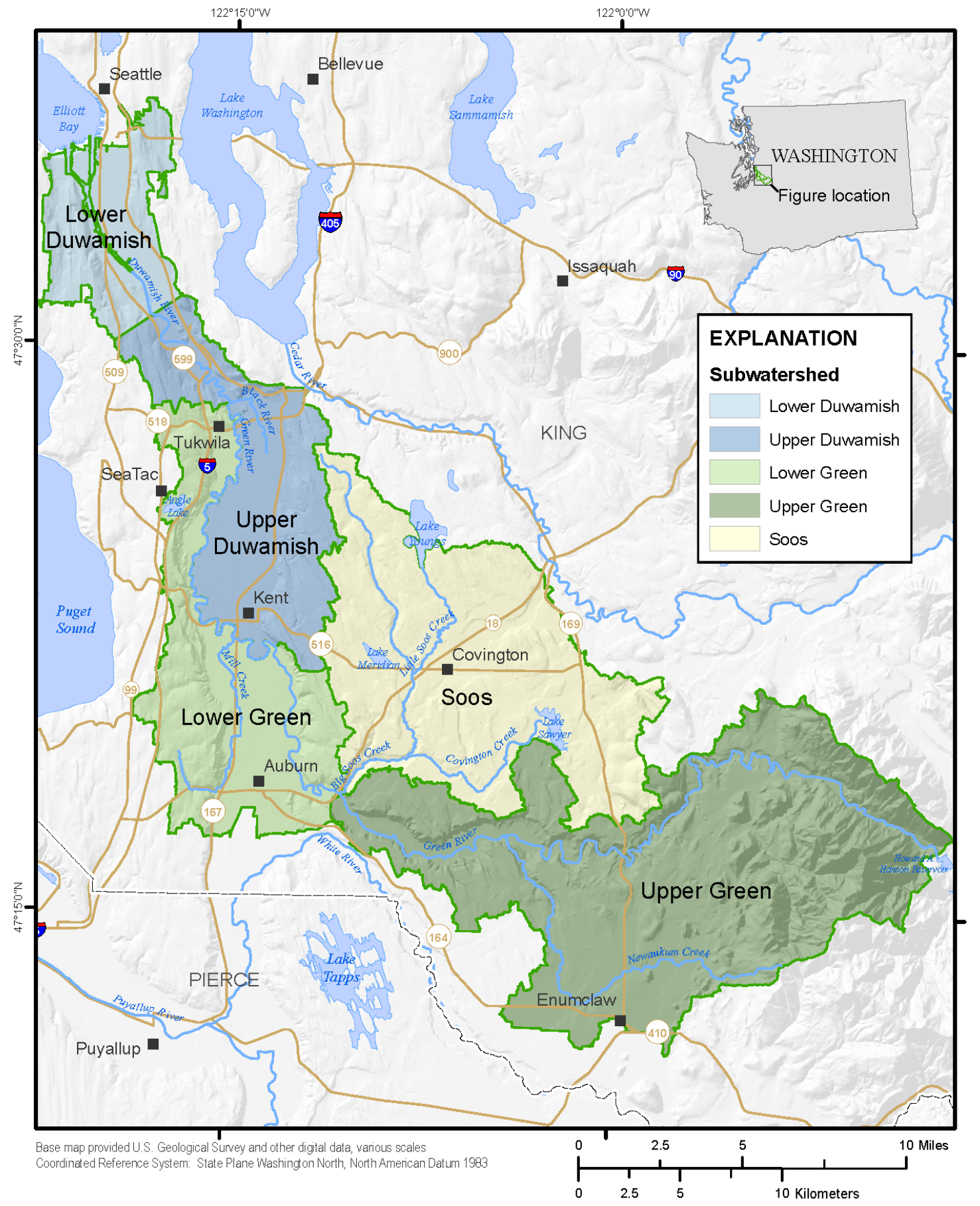

Figure 1. Map showing location of the Green-Duwamish River Watershed and subwatersheds, Washington. 
To support the implementation of the LDW cleanup plan, Ecology is leading source-control activities and a watershed-scale Pollutant Loading Assessment (PLA) to identify sources of sediment recontamination adjacent to and upstream of the LDW (Washington Department of Ecology, 2014). The objective of the PLA is to improve the understanding of water, sediment, and fish tissue quality, and the overall health of the Green-Duwamish watershed. To date, identified sources of contamination include stormwater, suspended sediment, and atmospheric deposition, and control activities have been implemented for stormwater and suspended sediment. Another potential source of contaminants to the Green-Duwamish River is from groundwater, which can move through contaminated soils and transport pollutants to the river through direct groundwater discharge. Groundwater direction and flow has been modeled in the LDW (Fabritz and others, 1998), but is not well understood throughout the remainder of the watershed. The Green-Duwamish watershed geology, like other adjacent watersheds, is underlain by a northwest-thickening sequence of unconsolidated glacial (till and outwash) and interglacial (fluvial, lacustrine, and mudflow) deposits. Sedimentary and volcanic bedrock units underlie the unconsolidated deposits (Welch and others, 2015).

Ecology is developing watershed models to simulate the hydrology of the basin. In the models the basin is divided into four subwatersheds: Duwamish, Lower Green, Soos, and Upper Green (fig. 1). The upper boundary of the Green-Duwamish watershed in the models is the Howard Hanson Dam, located at river kilometer (RKM) 103, which regulates river discharge primarily for flood control. The portion of the watershed above the Howard Hanson Dam, which is primarily forested land, is not included in Ecology's models and therefore, also is not included in the scope of this report. The Upper Green subwatershed is the most upstream subwatershed from the downstream side of the Howard Hanson Dam (RKM 103) to the confluence of the Green River with Big Soos Creek (RKM 55) and includes the city of Enumclaw. The Lower Green subwatershed extends from the confluence with Big Soos Creek (RKM 55) to the confluence with the Black River where the river changes names from Green to Duwamish (approximately RKM 18). The Lower Green subwatershed includes land on both sides of the river from RKM 55 to 44; however, from RKM 44 to 18 it contains land only on the west side of the river. The Lower Green subwatershed includes the cities of SeaTac and Auburn. The Soos subwatershed is unique because it does not include the Green-Duwamish River, but drains Big Soos Creek, a tributary to the Green River, and includes the cities of Covington and Maple Valley. The Duwamish subwatershed includes the LDW (RKM 0 to 8) and land on the eastern side of the river up to about RKM 44, including the cities of Tukwila and Kent. For this report, the Duwamish subwatershed was subdivided into the Lower Duwamish, containing the LDW, and the Upper Duwamish, containing the remaining area on both sides of the river to RKM 18 (the Black River confluence) and land only on the east side of the river from RKM 18 to 44. The reason for this subdivision was that, because of known sources of contamination, the groundwater quality in the Lower Duwamish is likely different from the rest of the Duwamish subwatershed. The lower boundary of the watershed is at RKM 0 where the river enters Elliott Bay just beyond Harbor Island in Seattle, Washington (fig. 1).

\section{Purpose and Scope}

This report summarizes results of a preliminary data compilation of groundwater quality data in the Green-Duwamish watershed. The sources of existing data were the USGS's National Water Information System, Ecology's Environmental Information Management System, and a compilation of several studies by Leidos, a scientific research company. The water quality parameters of interest were polychlorinated biphenyl (PCB) Aroclors and congeners, phthalates, carcinogenic polycyclic aromatic hydrocarbons (cPAHs), arsenic, copper, and zinc. Results were grouped into the four subwatersheds delineated in Ecology's hydrology models: Duwamish, Lower Green, Soos, and Upper Green. The 
purpose is to provide information on available groundwater concentration data to aid Ecology select appropriate chemical concentration values as input into their watershed models, which will estimate chemical loading from groundwater. Understanding the sources and contributions of contaminants in the Green-Duwamish watershed will support the ongoing cleanup activities in the LDW.

\section{Dataset Compilation and Analysis Methods}

Data were compiled from three data sources. The first source was the Green-Duwamish watershed PLA Database, created by Leidos, which included chemical concentrations in surface water, groundwater, and soil samples, and included other variables and metadata. The USGS extracted all groundwater data from this database. The second source was the USGS National Water Information System (NWIS) database, where a data retrieval was performed on February 19, 2019, for the parameters of interest in groundwater. The third source was Ecology's Environmental Information Management (EIM) database. Data was retrieved from EIM on February 21, 2019, for the parameters of interest in groundwater. All three data sources could not simply be added together because there was a significant amount of duplicated data, primarily due to the PLA database containing EIM data. Once duplicate data were identified and removed, the three data sources were merged into one file. The following attributes were retained: latitude, longitude, sample date, sample time, result, result unit, final qualifier, parameter name, detection limit, detection type, detection (yes or no), study name, and source of data. There were many results that did not include information for some of these attributes, and those fields were left blank. Other data attributes were discarded throughout the process of consolidating the multiple datasets.

The six water quality parameters of interest resulted in 14 unique parameters due to their different chemical forms, methods of collection, and methods of laboratory analysis. Arsenic was reported four ways: arsenic (unfiltered sample), dissolved arsenic (filtered sample), inorganic arsenic (unfiltered sample), and arsenic III. PCBs were reported three ways: sum of Aroclors, sum of 209 congeners, and sum of 209 congeners as toxic equivalents (TEQ). Copper samples included unfiltered samples and filtered samples. cPAHs were a sum of the following seven compounds: benz[a]anthracene, chrysene, benzo[a]pyrene, indeno[1,2,3-cd]pyrene, dibenz[a,h]anthracene, benzo[b]fluoranthene, benzo[j]fluoranthene, and benzo[k]fluoranthene. cPAHs were reported as TEQ in both unfiltered and filtered forms. Zinc was reported as zinc (unfiltered) and dissolved zinc (filtered). Phthalates were represented by an indicator compound, bis(2-ethylhexyl)phthalate (BEHP). Each parameter is unique and should not be used interchangeably or summed with other parameters.

After the data was consolidated into a single dataset, spatial attributes were computed using the geospatial software ESRI ArcGIS version 10.6.1. The subwatershed of each data point was labeled by overlaying the locations of the results with the delineated subwatersheds. The closest distance between each data point and the Green/Duwamish River was measured in each subwatershed (except for the Soos subwatershed because neither the Green nor Duwamish Rivers are within this subwatershed). The river changes names from the Green River to the Duwamish River at the Black River confluence at RKM 18.

Summary statistics including minimum, median, mean, and maximum concentration were calculated for each of the 14 parameters for each of the four subwatersheds (Upper Green, Soos, Lower Green, Upper Duwamish, and Lower Duwamish). The analysis was done for four sets of data:

1. All data;

2. Only data from wells within 1000 feet of the Green-Duwamish River;

3. Only non-censored data (for example, above the reporting limit); and

4. Only non-censored data from wells within 1000 feet of the Green-Duwamish River. 
Samples within 1,000 $\mathrm{ft}$ of the river were analyzed additionally because this area is assumed to have the greatest potential to affect water quality in the river. Many of the results were censored, for example, because they were below the laboratory reporting limit. The methods of Helsel (2005) were used to address uncertainties in reporting summary statistics on datasets with censored data. For datasets with less than 50 percent censored data, the Kaplan-Meier method was used. For datasets with 50-80 percent censored data, the Regression on Order Statistics (ROS; for datasets with less than 50 observations) or maximum likelihood estimation (MLE; for datasets with 50 observations or more) was used. For datasets with more than 80 percent censored data, only the maximum concentration is reported. Summary statistics were calculated using the "cenfit," "cenros," and "cenmle" functions for KaplanMeier, ROS, and MLE, respectively, from the NADA package (Lee, 2017) in R statistical computing environment (R Core Team, 2019).

\section{Groundwater Quality in the Green-Duwamish Watershed}

The total number of groundwater quality results compiled in this report was 22,520 from a total of 1,607 sites sampled from October 3, 1962 to May 24, 2018. All but 296 results were in the Duwamish subwatershed. Very few results were available for the six parameters of interest in the other three subwatersheds. No PCB or phthalate data in groundwater were found in the Soos and Upper Green subwatersheds. Results for phthalates were also absent in the Lower Green subwatershed. No results for cPAHs were found in the Upper Green watershed. The data that were compiled for each parameter are summarized in more detail below.

\section{Arsenic}

Samples analyzed for arsenic were available in all four subwatersheds and included four unique parameters: unfiltered samples of "arsenic," "inorganic arsenic," "arsenic III," and filtered samples "dissolved arsenic" (fig. 2). For this and subsequent figures, each parameter is identified by a unique symbol and the symbols are located at wells with analytical results. Those sampling sites with results for more than one type of parameter result have overlapping parameter symbols. Inorganic arsenic includes the speciations arsenic III and arsenic V. The unfiltered samples labeled "arsenic" includes all speciations in the whole water sample. Likewise, "dissolved arsenic" includes all speciations of arsenic in the filtered sample.

There were 5,271 samples analyzed for arsenic, and 12 percent of those were below detection (table 1). Results were available for all subwatersheds except the Upper Green. However, most arsenic data were in the Duwamish subwatershed, with the majority (71 percent) of those within the Lower Duwamish. Only 14 results were in the Lower Green subwatershed and 1 was in the Soos subwatershed. Detected concentrations ranged from 0.05 to 274,000 micrograms per liter $(\mu \mathrm{g} / \mathrm{L})$. The maximum concentration $(274,000 \mu \mathrm{g} / \mathrm{L})$ was in the Lower Duwamish. The Lower Duwamish had higher median and mean concentrations than the Upper Duwamish and Lower Green subwatersheds. Across the watershed, median arsenic concentrations were similar, between 3.55 and $4.9 \mu \mathrm{g} / \mathrm{L}$, regardless of the method used (statistically estimated to account for censored data, using only detected values for all samples, and only for samples within 1,000 feet of the Green-Duwamish River) (table 1). There were 1,897 dissolved arsenic results, and 15 percent of those were below detection (table 1). Most (91 percent) of the dissolved arsenic results were in the Lower Duwamish. However, there was at least one result in each subwatershed. Detected concentrations ranged from 0.0778 to $285,000 \mu \mathrm{g} / \mathrm{L}$. Similar to total arsenic, the maximum concentration was in the Lower Duwamish, resulting in a higher mean 
concentration in the Lower Duwamish $(900 \mu \mathrm{g} / \mathrm{L})$ than in the other subwatersheds (below detection limit $[12.3 \mu \mathrm{g} / \mathrm{L}])$.

There were 32 arsenic III results, none of which were non-detections (table 1). All results were in the Lower Duwamish. Concentrations ranged from 3.98 to $2,720 \mu \mathrm{g} / \mathrm{L}$, with a median and mean of 112 and $500 \mu \mathrm{g} / \mathrm{L}$, respectively.

There were 15 inorganic arsenic results, none of which were non-detections (table 1). Inorganic arsenic results were only available in the Lower Duwamish. Concentrations ranged from 6.88 to 2,930 $\mu \mathrm{g} / \mathrm{L}$, with a median and mean of 147 and $624 \mu \mathrm{g} / \mathrm{L}$, respectively. 


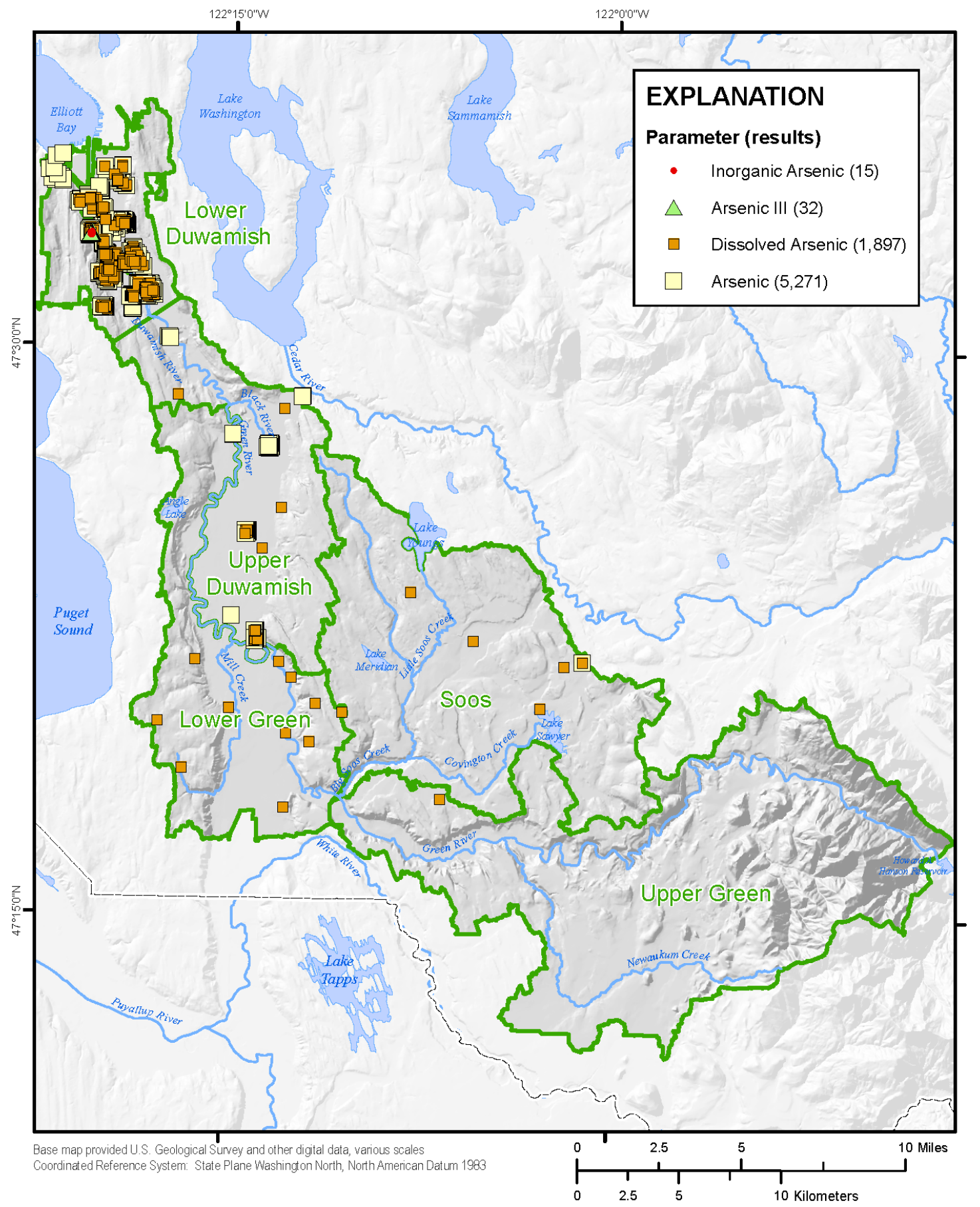

Figure 2. Map showing distribution of groundwater sites with arsenic data, Green-Duwamish watershed, Washington. 
Table 1. Summary of data for arsenic in groundwater samples in the Green-Duwamish watershed, Washington.

[All: All results. $<\mathbf{1 , 0 0 0 ~ f t : ~ R e s u l t s ~ f r o m ~ w e l l s ~ w i t h i n ~ 1 , 0 0 0 ~ f e e t ~ o f ~ t h e ~ G r e e n - D u w a m i s h ~ R i v e r . ~ A b b r e v i a t i o n s : ~ B D , ~ b e l o w ~ d e t e c t i o n ; ~ K M , ~ K a p l a n - M e i e r ; ~ N A , ~}$ not applicable; ROS, Regression on Order Statistics; $\mu \mathrm{g} / \mathrm{L}$, micrograms per liter.]

\begin{tabular}{|c|c|c|c|c|c|c|c|c|c|c|c|}
\hline \multirow[t]{2}{*}{ Parameter } & \multicolumn{2}{|c|}{ Entire watershed } & \multicolumn{4}{|c|}{ Duwamish subwatershed } & \multicolumn{2}{|c|}{$\begin{array}{l}\text { Lower Green } \\
\text { subwatershed }\end{array}$} & \multirow{2}{*}{$\begin{array}{c}\begin{array}{c}\text { Soos } \\
\text { subwatershed }\end{array} \\
\text { All } \\
\end{array}$} & \multicolumn{2}{|c|}{$\begin{array}{l}\text { Upper Green } \\
\text { subwatershed }\end{array}$} \\
\hline & All & $<1,000 \mathrm{ft}$ & All & $<1,000 \mathrm{ft}$ & All & $<1,000 \mathrm{ft}$ & All & $<1,000 \mathrm{ft}$ & & All & $<1,000 \mathrm{ft}$ \\
\hline \multicolumn{12}{|c|}{ Unfiltered arsenic } \\
\hline Number of results & 5,271 & 2,024 & 4,003 & 2,002 & 1,253 & 8 & 14 & 14 & 1 & 0 & 0 \\
\hline $\begin{array}{l}\text { Percent of results below } \\
\text { detection }\end{array}$ & 12 & 14 & 15 & 14 & 3 & 75 & 0 & 0 & 0 & NA & NA \\
\hline $\begin{array}{l}\text { Summary statistics estimator } \\
\text { method }\end{array}$ & KM & KM & KM & KM & KM & ROS & NA & NA & NA & NA & NA \\
\hline Minimum $(\mu \mathrm{g} / \mathrm{L})$ & $\mathrm{BD}$ & $\mathrm{BD}$ & $\mathrm{BD}$ & $\mathrm{BD}$ & $\mathrm{BD}$ & $\mathrm{BD}$ & 4.21 & 4.21 & NA & NA & NA \\
\hline $\begin{array}{l}\text { Minimum }(\mu \mathrm{g} / \mathrm{L}) \text { of detected } \\
\text { values }\end{array}$ & 0.05 & 0.137 & 0.05 & 0.137 & 0.4 & 2.8 & 4.21 & 4.21 & NA & NA & NA \\
\hline $\begin{array}{l}\text { Median }(\mu \mathrm{g} / \mathrm{L}) \text { using estimator } \\
\text { method }\end{array}$ & 3.7 & 3.55 & 2.7 & 3.5 & 7.41 & 0.838 & NA & NA & NA & NA & NA \\
\hline $\begin{array}{l}\text { Median }(\mu \mathrm{g} / \mathrm{L}) \text { of detected } \\
\text { values }\end{array}$ & 4.9 & 4.78 & 3.95 & 4.70 & 7.7 & NA & 19.3 & 19.3 & NA & NA & NA \\
\hline $\begin{array}{l}\text { Mean }(\mu \mathrm{g} / \mathrm{L}) \text { using estimator } \\
\text { method }\end{array}$ & 565 & 1,354 & 740 & 1,369 & 11.5 & 1.88 & NA & NA & NA & NA & NA \\
\hline Mean $(\mu \mathrm{g} / \mathrm{L})$ of detected values & 645 & 1,572 & 875 & 1,586 & 11.7 & 5.65 & 16.8 & 16.8 & NA & NA & NA \\
\hline Maximum $(\mu \mathrm{g} / \mathrm{L})$ & $\begin{array}{c}274,000 \\
11-7-\end{array}$ & $\begin{array}{r}274,000 \\
11-7-\end{array}$ & $\begin{array}{r}274,000 \\
11-7-\end{array}$ & $\begin{array}{r}274,000 \\
11-7-\end{array}$ & $\begin{array}{l}148 \\
1-16-\end{array}$ & $\begin{array}{l}8.5 \\
5-30-\end{array}$ & $\begin{array}{l}22.6 \\
6-12-\end{array}$ & $\begin{array}{l}22.6 \\
6-12-\end{array}$ & & NA & NA \\
\hline $\begin{array}{l}\text { Oldest sampling date } \\
\text { Most recent sampling date }\end{array}$ & $\begin{array}{l}1988 \\
5-24- \\
2018 \\
\end{array}$ & $\begin{array}{l}1988 \\
2-14- \\
2018 \\
\end{array}$ & $\begin{array}{l}1988 \\
5-24- \\
2018 \\
\end{array}$ & $\begin{array}{l}1988 \\
2-14- \\
2018 \\
\end{array}$ & $\begin{array}{l}2006 \\
5-1- \\
2018 \\
\end{array}$ & $\begin{array}{l}2006 \\
6-18- \\
2015 \\
\end{array}$ & $\begin{array}{l}2008 \\
3-18- \\
2009 \\
\end{array}$ & $\begin{array}{l}2008 \\
3-18- \\
2009 \\
\end{array}$ & $\begin{array}{l}3-8-2012 \\
3-8-2012\end{array}$ & $\begin{array}{l}\text { NA } \\
\text { NA }\end{array}$ & $\begin{array}{l}\text { NA } \\
\text { NA }\end{array}$ \\
\hline \multicolumn{12}{|c|}{ Dissolved arsenic } \\
\hline Number of results & 1,897 & 1,030 & 1,732 & 1,017 & 144 & 10 & 13 & 3 & 7 & 1 & 0 \\
\hline $\begin{array}{l}\text { Percent of results below } \\
\text { detection } \\
\text { Summary statistics estimator }\end{array}$ & 15 & 15 & 15 & 14 & 11 & 90 & 31 & 67 & 43 & 100 & NA \\
\hline method & KM & KM & KM & KM & KM & NA & KM & NA & KM & NA & NA \\
\hline Minimum $(\mu \mathrm{g} / \mathrm{L})$ & $\mathrm{BD}$ & $\mathrm{BD}$ & $\mathrm{BD}$ & $\mathrm{BD}$ & $\mathrm{BD}$ & $\mathrm{BD}$ & $\mathrm{BD}$ & $\mathrm{BD}$ & $\mathrm{BD}$ & $\mathrm{BD}$ & NA \\
\hline $\begin{array}{l}\text { Minimum }(\mu \mathrm{g} / \mathrm{L}) \text { of detected } \\
\text { values }\end{array}$ & 0.078 & 0.078 & 0.078 & 0.078 & 0.5 & NA & 2 & NA & 0.2 & $\mathrm{BD}$ & NA \\
\hline $\begin{array}{l}\text { Median }(\mu \mathrm{g} / \mathrm{L}) \text { using estimator } \\
\text { method }\end{array}$ & 3.7 & 3.7 & 3.6 & 3.79 & 6.2 & NA & 3 & NA & 0.2 & $\mathrm{BD}$ & NA \\
\hline Median $(\mu \mathrm{g} / \mathrm{L})$ of detected & 5.08 & 5.02 & 5 & 5.07 & 7.87 & NA & 3 & NA & 1.88 & $\mathrm{BD}$ & NA \\
\hline
\end{tabular}




\begin{tabular}{|c|c|c|c|c|c|c|c|c|c|c|c|}
\hline \multirow[t]{2}{*}{ Parameter } & \multicolumn{2}{|c|}{ Entire watershed } & \multicolumn{4}{|c|}{ Duwamish subwatershed } & \multicolumn{2}{|c|}{$\begin{array}{l}\text { Lower Green } \\
\text { subwatershed }\end{array}$} & \multirow{2}{*}{$\begin{array}{c}\begin{array}{c}\text { Soos } \\
\text { subwatershed }\end{array} \\
\text { All }\end{array}$} & \multicolumn{2}{|c|}{$\begin{array}{l}\text { Upper Green } \\
\text { subwatershed }\end{array}$} \\
\hline & All & $<1,000 \mathrm{ft}$ & All & $<1,000 \mathrm{ft}$ & All & $<1,000 \mathrm{ft}$ & All & $<1,000 \mathrm{ft}$ & & All & $<1,000 \mathrm{ft}$ \\
\hline values & & & & & & & & & & & \\
\hline $\begin{array}{l}\text { Mean }(\mu \mathrm{g} / \mathrm{L}) \text { using estimator } \\
\text { method }\end{array}$ & 823 & 1,372 & 900 & 1,390 & 12.3 & NA & 2.92 & NA & 1.08 & $\mathrm{BD}$ & NA \\
\hline Mean $(\mu \mathrm{g} / \mathrm{L})$ of detected values & 972 & 1,606 & 1064 & 1,610 & 13.7 & NA & 3.33 & NA & 1.74 & $\mathrm{BD}$ & NA \\
\hline $\begin{array}{l}\text { Maximum }(\mu \mathrm{g} / \mathrm{L}) \\
\text { Oldest sampling date } \\
\text { Most recent sampling date }\end{array}$ & $\begin{array}{l}285,000 \\
3-2- \\
1988 \\
8-17- \\
2017 \\
\end{array}$ & $\begin{array}{l}285,000 \\
3-4- \\
1988 \\
10-1- \\
2016 \\
\end{array}$ & $\begin{array}{l}285,000 \\
1-30- \\
1996 \\
8-17- \\
2017 \\
\end{array}$ & $\begin{array}{l}285,000 \\
1-30- \\
1996 \\
10-1- \\
2016 \\
\end{array}$ & $\begin{array}{l}100 \\
3-9- \\
1988 \\
4-25- \\
2017 \\
\end{array}$ & $\begin{array}{l}2.1 \\
5-30- \\
2006 \\
8-29- \\
2006 \\
\end{array}$ & $\begin{array}{c}7 \\
3-2- \\
1988 \\
3-17- \\
1988 \\
\end{array}$ & $\begin{array}{c}3 \\
3-4- \\
1988 \\
3-11- \\
1988 \\
\end{array}$ & $\begin{array}{l}3 \\
3-14-1988 \\
11-29-2016\end{array}$ & $\begin{array}{r}\text { BD } \\
3-8- \\
1988 \\
3-8- \\
1988 \\
\end{array}$ & $\begin{array}{l}\text { NA } \\
\text { NA } \\
\text { NA }\end{array}$ \\
\hline \multicolumn{12}{|c|}{ Arsenic III } \\
\hline \multirow{3}{*}{$\begin{array}{l}\text { Number of results } \\
\text { Percent of results below } \\
\text { detection } \\
\text { Summary statistics estimator } \\
\text { method }\end{array}$} & 32 & 30 & 32 & 30 & 0 & 0 & 0 & 0 & 0 & 0 & 0 \\
\hline & 0 & 0 & 0 & 0 & NA & NA & NA & NA & NA & NA & NA \\
\hline & NA & NA & NA & NA & NA & NA & NA & NA & NA & NA & NA \\
\hline \multirow{3}{*}{$\begin{array}{l}\text { Minimum }(\mu \mathrm{g} / \mathrm{L}) \\
\text { Minimum }(\mu \mathrm{g} / \mathrm{L}) \text { of detected } \\
\text { values } \\
\text { Median }(\mu \mathrm{g} / \mathrm{L}) \text { using estimator } \\
\text { method }\end{array}$} & 3.98 & 3.98 & 3.98 & 3.98 & NA & NA & NA & NA & NA & NA & NA \\
\hline & 3.98 & 3.98 & 3.98 & 3.98 & NA & NA & NA & NA & NA & NA & NA \\
\hline & NA & NA & NA & NA & NA & NA & NA & NA & NA & NA & NA \\
\hline $\begin{array}{l}\text { Median }(\mu \mathrm{g} / \mathrm{L}) \text { of detected } \\
\text { values }\end{array}$ & 112 & 43.80 & 112 & 43.80 & NA & NA & NA & NA & NA & NA & NA \\
\hline $\begin{array}{l}\text { Mean }(\mu \mathrm{g} / \mathrm{L}) \text { using estimator } \\
\text { method }\end{array}$ & NA & NA & NA & NA & NA & NA & NA & NA & NA & NA & NA \\
\hline Mean $(\mu \mathrm{g} / \mathrm{L})$ of detected values & 550 & 566 & 550 & 566 & NA & NA & NA & NA & NA & NA & NA \\
\hline Maximum $(\mu \mathrm{g} / \mathrm{L})$ & 2,720 & 2,720 & 2,720 & 2,720 & NA & NA & NA & NA & NA & NA & NA \\
\hline Oldest sampling date & 2009 & 2009 & 2009 & 2009 & NA & NA & NA & NA & NA & NA & NA \\
\hline Most recent sampling date & $\begin{array}{l}3-26- \\
2009 \\
\end{array}$ & $\begin{array}{l}3-26- \\
2009 \\
\end{array}$ & $\begin{array}{l}3-26- \\
2009 \\
\end{array}$ & $\begin{array}{l}3-26- \\
2009 \\
\end{array}$ & NA & NA & NA & NA & NA & NA & NA \\
\hline \multicolumn{12}{|c|}{ Inorganic arsenic } \\
\hline \multirow{3}{*}{$\begin{array}{l}\text { Number of results } \\
\text { Percent of results below } \\
\text { detection } \\
\text { Summary statistics estimator } \\
\text { method }\end{array}$} & 15 & 14 & 15 & 14 & 0 & 0 & 0 & 0 & 0 & 0 & 0 \\
\hline & 0 & 0 & 0 & 0 & NA & NA & NA & NA & NA & NA & NA \\
\hline & NA & NA & NA & NA & NA & NA & NA & NA & NA & NA & NA \\
\hline
\end{tabular}




\begin{tabular}{|c|c|c|c|c|c|c|c|c|c|c|c|}
\hline \multirow{2}{*}{ Parameter } & \multicolumn{2}{|c|}{ Entire watershed } & \multicolumn{4}{|c|}{ Duwamish subwatershed } & \multicolumn{2}{|c|}{$\begin{array}{l}\text { Lower Green } \\
\text { subwatershed }\end{array}$} & \multirow{2}{*}{$\begin{array}{c}\text { Soos } \\
\text { subwatershed } \\
\text { All }\end{array}$} & \multicolumn{2}{|c|}{$\begin{array}{l}\text { Upper Green } \\
\text { subwatershed }\end{array}$} \\
\hline & All & $<1,000 \mathrm{ft}$ & All & $<1,000 \mathrm{ft}$ & All & $<1,000 \mathrm{ft}$ & All & $<1,000 \mathrm{ft}$ & & All & $<1,000 \mathrm{ft}$ \\
\hline Minimum $(\mu \mathrm{g} / \mathrm{L})$ & 6.88 & 6.88 & 6.88 & 6.88 & NA & NA & NA & NA & NA & NA & NA \\
\hline $\begin{array}{l}\text { Minimum }(\mu \mathrm{g} / \mathrm{L}) \text { of detected } \\
\text { values }\end{array}$ & 6.88 & 6.88 & 6.88 & 6.88 & NA & NA & NA & NA & NA & NA & NA \\
\hline $\begin{array}{l}\text { Median }(\mu \mathrm{g} / \mathrm{L}) \text { using estimator } \\
\text { method } \\
\text { Median }(\mu \mathrm{g} / \mathrm{L}) \text { of detected }\end{array}$ & NA & NA & NA & NA & NA & NA & NA & NA & NA & NA & NA \\
\hline $\begin{array}{l}\text { Median }(\mu \mathrm{g} / \mathrm{L}) \text { of detected } \\
\text { values }\end{array}$ & 147 & 98.40 & 147 & 98.40 & NA & NA & NA & NA & NA & NA & NA \\
\hline $\begin{array}{l}\text { Mean }(\mu \mathrm{g} / \mathrm{L}) \text { using estimator } \\
\text { method }\end{array}$ & NA & NA & NA & NA & NA & NA & NA & NA & NA & NA & NA \\
\hline Mean $(\mu \mathrm{g} / \mathrm{L})$ of detected values & 624 & 644 & 624 & 644 & NA & NA & NA & NA & NA & NA & NA \\
\hline Maximum $(\mu \mathrm{g} / \mathrm{L})$ & $\begin{array}{l}2,930 \\
3-23-\end{array}$ & $\begin{array}{l}2,930 \\
3-23-\end{array}$ & $\begin{array}{l}2,930 \\
3-23-\end{array}$ & $\begin{array}{l}2,930 \\
3-23-\end{array}$ & NA & NA & NA & NA & NA & NA & NA \\
\hline Oldest sampling date & 2009 & 2009 & 2009 & 2009 & NA & NA & NA & NA & NA & NA & NA \\
\hline Most recent sampling date & $\begin{array}{l}3-26- \\
2009\end{array}$ & $\begin{array}{l}3-26- \\
2009\end{array}$ & $\begin{array}{l}3-26- \\
2009\end{array}$ & $\begin{array}{l}3-26- \\
2009\end{array}$ & NA & NA & NA & NA & NA & NA & NA \\
\hline
\end{tabular}




\section{Copper}

Copper data were available in all four subwatersheds (fig. 3) and included two parameters: "copper" (in unfiltered samples) and "dissolved copper" (in filtered samples). There were 1,658 unfiltered copper results, of which 29 percent were below detection (table 2). Detected concentrations ranged from 0.05 to $1,790 \mu \mathrm{g} / \mathrm{L}$, and were limited to the Duwamish subwatershed, with the majority (98 percent) in the Lower Duwamish. Detected concentrations of unfiltered copper in groundwater from wells within 1,000 feet of the Green-Duwamish River ranged from 0.13 to $1,790 \mu \mathrm{g} / \mathrm{L}$. The unfiltered copper data from the Upper Duwamish was limited to 26 samples, with a maximum concentration of 3.6 $\mu \mathrm{g} / \mathrm{L}$. All 10 samples within 1,000 ft of the river were below detection. There were no available data for unfiltered copper in groundwater from the Lower Green, Soos, or Upper Green subwatersheds.

There were 1,331 results for dissolved copper in groundwater, and 49 percent were below detection (table 2). The detected concentrations ranged from 0.08 to $2,050 \mu \mathrm{g} / \mathrm{L}$. The majority (96 percent) of the dissolved copper results were from the Lower Duwamish. The median value in the Lower Duwamish was $0.60 \mu \mathrm{g} / \mathrm{L}$ using the Kaplan-Meier method and 1.90 using only detected values. Summary statistics for dissolved copper in groundwater from the wells within 1,000 $\mathrm{ft}$ of the river in the Lower Duwamish subwatershed were similar to those using all Lower Duwamish data. Like the arsenic data, maximum concentrations were highest in the Lower Duwamish as compared to the other subwatersheds (2,050 versus between 2 and $75 \mu \mathrm{g} / \mathrm{L}$, respectively; table 2). The most recent samples for dissolved copper in the Lower Green and Upper Green subwatersheds were collected in 1988. 


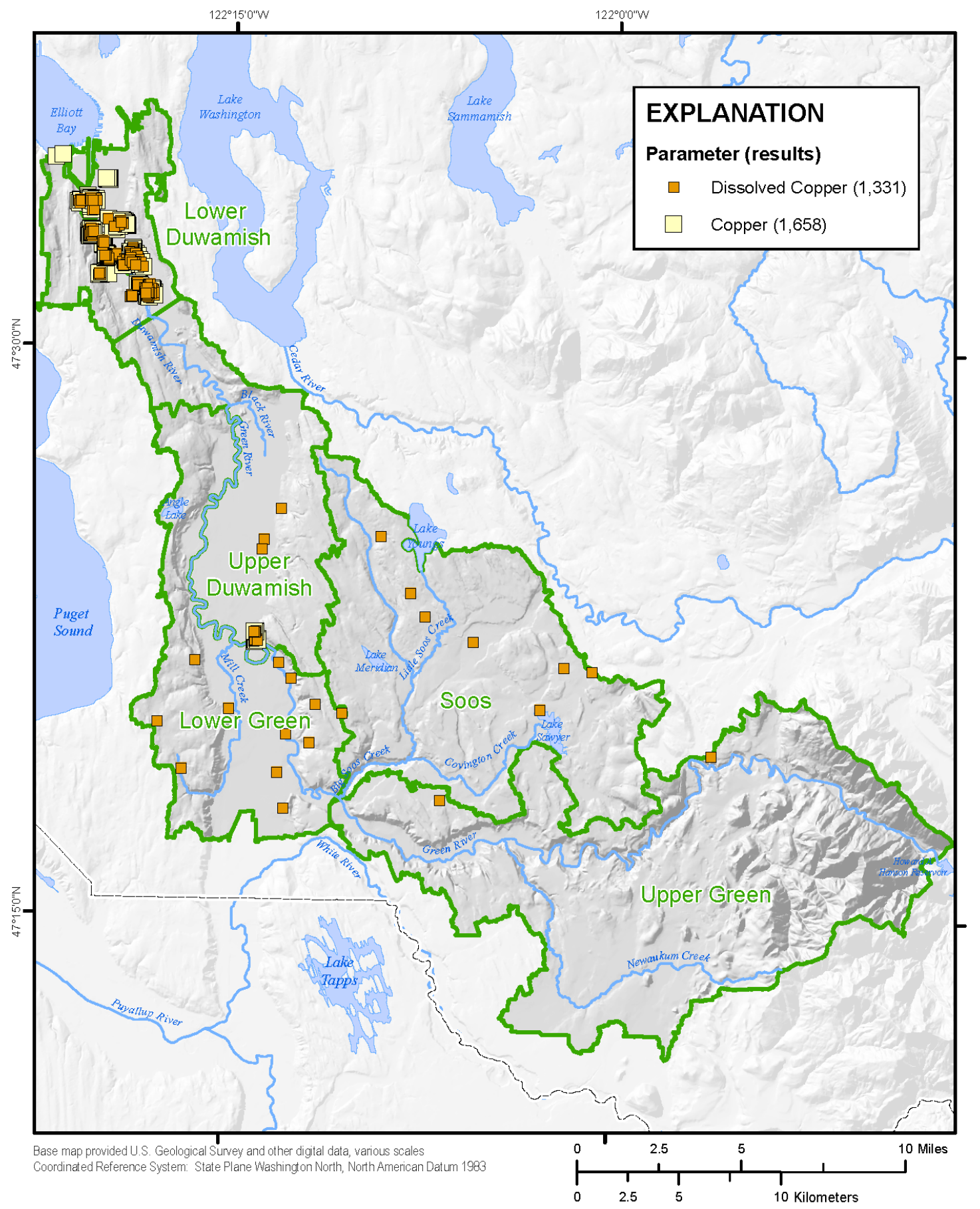

Figure 3. Map showing distribution of groundwater sites with copper data, Green-Duwamish watershed, Washington. 
Table 2. Summary of data for copper in groundwater samples in the Green-Duwamish watershed, Washington.

[All: All results. <1,000 ft: Results from wells within 1,000 feet of the Green-Duwamish River. Abbreviations: BD, below detection; KM, Kaplan-Meier; MLE, maximum likelihood estimation; NA, not applicable; ROS, Regression on Order Statistics; $\mu \mathrm{g} / \mathrm{L}$, micrograms per liter.]

\begin{tabular}{|c|c|c|c|c|c|c|c|c|c|c|c|}
\hline \multirow{3}{*}{ Parameter } & \multirow{2}{*}{\multicolumn{2}{|c|}{ Entire watershed }} & \multicolumn{4}{|c|}{ Duwamish subwatershed } & \multirow{2}{*}{\multicolumn{2}{|c|}{$\begin{array}{l}\text { Lower Green } \\
\text { subwatershed }\end{array}$}} & \multirow{3}{*}{$\begin{array}{c}\begin{array}{c}\text { Soos } \\
\text { subwatershed }\end{array} \\
\text { All }\end{array}$} & \multirow{2}{*}{\multicolumn{2}{|c|}{$\begin{array}{l}\text { Upper Green } \\
\text { subwatershed }\end{array}$}} \\
\hline & & & \multicolumn{2}{|c|}{ Lower Duwamish } & \multicolumn{2}{|c|}{ Upper Duwamish } & & & & & \\
\hline & All & $<1,000 \mathrm{ft}$ & All & $<1,000 \mathrm{ft}$ & All & $<1,000 \mathrm{ft}$ & All & $<1,000 \mathrm{ft}$ & & All & $\begin{array}{c}<1,000 \\
\mathrm{ft}\end{array}$ \\
\hline $\begin{array}{l}\text { Percent of results below } \\
\text { detection }\end{array}$ & 29 & 32 & 29 & 32 & 62 & 100 & NA & NA & NA & NA & NA \\
\hline $\begin{array}{l}\text { Summary statistics estimator } \\
\text { method }\end{array}$ & KM & KM & KM & KM & ROS & NA & NA & NA & NA & NA & NA \\
\hline $\begin{array}{l}\text { Minimum }(\mu \mathrm{g} / \mathrm{L}) \text { of detected } \\
\text { values }\end{array}$ & 0.05 & 0.13 & 0.05 & 0.13 & 0.6 & $\mathrm{BD}$ & NA & NA & NA & NA & NA \\
\hline $\begin{array}{l}\text { Median }(\mu \mathrm{g} / \mathrm{L}) \text { using estimator } \\
\text { method }\end{array}$ & 1.3 & 1.73 & 1.3 & 1.74 & 1.6 & $\mathrm{BD}$ & NA & NA & NA & NA & NA \\
\hline $\begin{array}{l}\text { Median }(\mu \mathrm{g} / \mathrm{L}) \text { of detected } \\
\text { values } \\
\text { Mean }(\mu \mathrm{g} / \mathrm{L}) \text { using estimator }\end{array}$ & 2.21 & 3.00 & 2.25 & 3.00 & 1.6 & $\mathrm{BD}$ & NA & NA & NA & NA & NA \\
\hline method & 11 & 13 & 11 & 13 & 1.73 & $\mathrm{BD}$ & NA & NA & NA & NA & NA \\
\hline \multicolumn{12}{|c|}{ Dissolved copper } \\
\hline Number of results & 1,331 & 944 & 1,280 & 931 & 25 & 10 & 15 & 3 & 9 & 2 & 0 \\
\hline $\begin{array}{l}\text { Percent of results below } \\
\text { detection }\end{array}$ & 49 & 53 & 48 & 53 & 84 & 100 & 40 & 33 & 44 & 50 & NA \\
\hline $\begin{array}{l}\text { Summary statistics estimator } \\
\text { method }\end{array}$ & KM & MLE & KM & MLE & NA & NA & KM & NA & KM & NA & NA \\
\hline Minimum $(\mu \mathrm{g} / \mathrm{L})$ & $\mathrm{BD}$ & $\mathrm{BD}$ & $\mathrm{BD}$ & $\mathrm{BD}$ & $\mathrm{BD}$ & NA & $\mathrm{BD}$ & $\mathrm{BD}$ & $\mathrm{BD}$ & $\mathrm{BD}$ & NA \\
\hline $\begin{array}{l}\text { Minimum }(\mu \mathrm{g} / \mathrm{L}) \text { of detected } \\
\text { values }\end{array}$ & 0.08 & 0.12 & 0.08 & 0.12 & 1 & NA & 1 & 2 & 1.1 & NA & NA \\
\hline $\begin{array}{l}\text { Median }(\mu \mathrm{g} / \mathrm{L}) \text { using estimator } \\
\text { method }\end{array}$ & 0.62 & 0.6 & 0.6 & 0.6 & NA & NA & 2 & NA & 3 & NA & NA \\
\hline
\end{tabular}




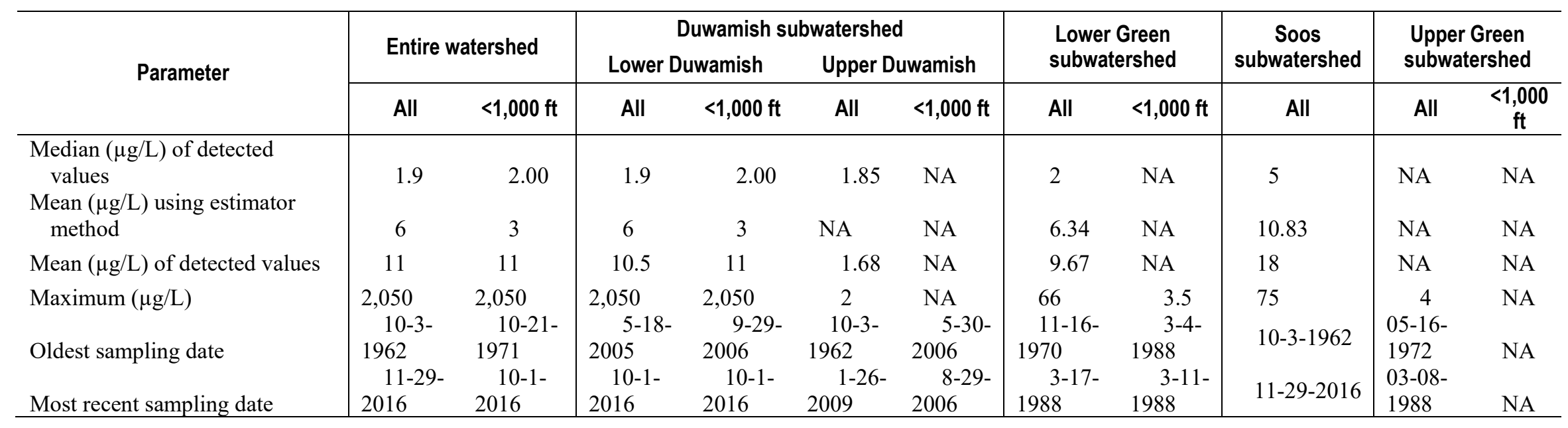




\section{Carcinogenic Polycyclic Aromatic Hydrocarbons}

Data for cPAHs as TEQ were available in three subwatersheds excluding the Upper Green. There were results for cPAHs as TEQ in unfiltered groundwater samples (cPAHs TEQ) and in filtered samples (dissolved cPAHs TEQ; fig. 4). There were 3,710 results for cPAHs as TEQ in unfiltered groundwater samples, and 85 percent were below detection. Detected concentrations ranged from 0.0029 to 85 micrograms TEQ per liter ( $\mu \mathrm{g}$ TEQ/L; table 3). Most (94 percent) of the cPAH data were in the Duwamish subwatershed and of those the majority (79 percent) were within the Lower Duwamish. There were 124 results for cPAHs in unfiltered groundwater samples from the Lower Green subwatershed, and 67 percent were below detection. There were 66 results for cPAHs from the Soos subwatershed, and 71 percent were below detection. There were no cPAH data available in the Upper Green subwatershed. Unlike arsenic and copper, the highest reported concentration $-85 \mu \mathrm{g} \mathrm{TEQ} / \mathrm{L}-$ was in the Upper Duwamish subwatershed and not in the Lower Duwamish. The minimum, median, and mean values of unfiltered cPAH as TEQ was also higher in the Upper Duwamish as compared to the other subwatersheds (table 3). The 12 dissolved cPAHs TEQ results were all within the Lower Duwamish and were below detection (table 3 ). 


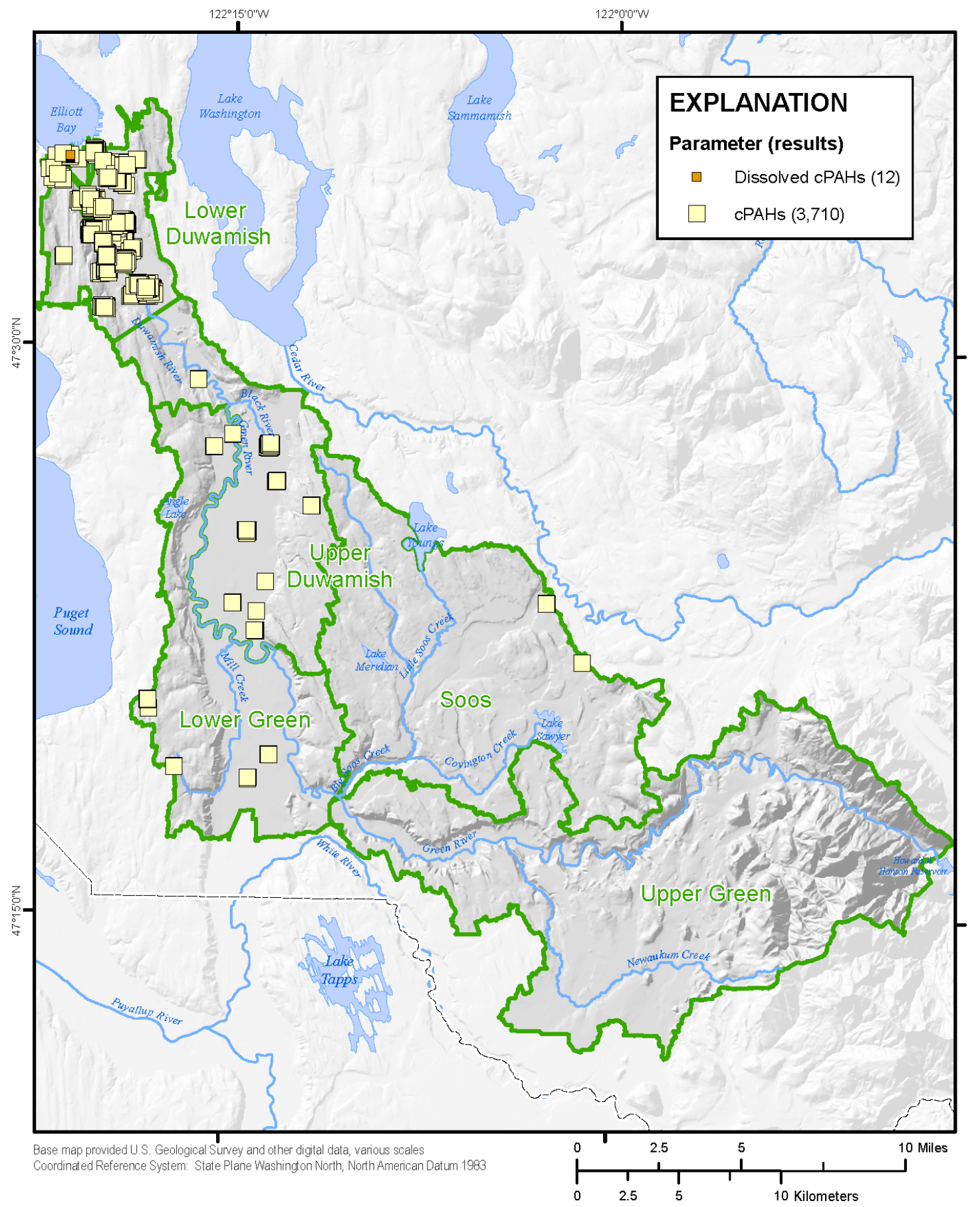

Figure 4. Map showing distribution of groundwater sites with polycyclic aromatic hydrocarbons (cPAH) data, Green-Duwamish watershed, Washington. 
Table 3. Summary of data for carcinogenic polycyclic aromatic hydrocarbons (cPAHs) in toxic equivalents (TEQ) in groundwater samples in the Green-Duwamish watershed, Washington.

[All: All results. $<\mathbf{1 , 0 0 0 ~ f t : ~ R e s u l t s ~ f r o m ~ w e l l s ~ w i t h i n ~ 1 , 0 0 0 ~ f e e t ~ o f ~ t h e ~ G r e e n - D u w a m i s h ~ R i v e r . ~ A b b r e v i a t i o n s : ~ B D , ~ b e l o w ~ d e t e c t i o n ; ~ M L E , ~ m a x i m u m ~}$ likelihood estimation; NA, not applicable; $\mu \mathrm{g} \mathrm{TEQ/L,} \mathrm{micrograms} \mathrm{toxic} \mathrm{equivalents} \mathrm{per} \mathrm{liter.]}$

\begin{tabular}{|c|c|c|c|c|c|c|c|c|c|c|c|}
\hline \multirow{3}{*}{ Parameter } & \multirow{2}{*}{\multicolumn{2}{|c|}{ Entire watershed }} & \multicolumn{4}{|c|}{ Duwamish subwatershed } & \multirow{2}{*}{\multicolumn{2}{|c|}{$\begin{array}{l}\text { Lower Green } \\
\text { subwatershed }\end{array}$}} & \multirow{3}{*}{$\begin{array}{c}\begin{array}{c}\text { Soos } \\
\text { subwatershed }\end{array} \\
\text { All } \\
\end{array}$} & \multirow{2}{*}{\multicolumn{2}{|c|}{$\begin{array}{l}\text { Upper Green } \\
\text { subwatershed }\end{array}$}} \\
\hline & & & \multicolumn{2}{|c|}{ Lower Duwamish } & \multicolumn{2}{|c|}{ Upper Duwamish } & & & & & \\
\hline & All & $<1,000 \mathrm{ft}$ & All & $<1,000 \mathrm{ft}$ & All & $\begin{array}{c}<1,000 \\
\mathrm{ft}\end{array}$ & All & $<1,000 \mathrm{ft}$ & & All & $<1,000 \mathrm{ft}$ \\
\hline \multicolumn{12}{|l|}{ Unfiltered cPAHs } \\
\hline Percent of results below detection & 85 & 86 & 86 & 86 & 89 & 100 & 67 & 88 & 71 & NA & NA \\
\hline Summary statistics estimator method & NA & NA & NA & NA & NA & NA & MLE & NA & MLE & NA & NA \\
\hline Minimum $(\mu \mathrm{g}$ TEQ/L) & $\mathrm{BD}$ & $\mathrm{BD}$ & $\mathrm{BD}$ & $\mathrm{BD}$ & $\mathrm{BD}$ & $\mathrm{BD}$ & $\mathrm{BD}$ & $\mathrm{BD}$ & $\mathrm{BD}$ & NA & NA \\
\hline $\begin{array}{l}\text { Minimum ( } \mu \mathrm{g} \text { TEQ/L) of detected } \\
\text { values } \\
\text { Median (ug TEO/L) using estimator }\end{array}$ & 0.0029 & 0.0037 & 0.0029 & 0.004 & 0.0338 & $\mathrm{BD}$ & 0.0079 & 0.022 & 0.0167 & NA & NA \\
\hline $\begin{array}{l}\text { Median }(\mu \mathrm{g} / E Q / L) \text { using estimator } \\
\text { method } \\
\text { Median }(\mu \mathrm{g} T E Q / L) \text { of detected }\end{array}$ & NA & NA & NA & NA & NA & $\mathrm{BD}$ & 0.0087 & NA & 0.0044 & NA & NA \\
\hline $\begin{array}{l}\text { values } \\
\text { Mean }(\mu \mathrm{g} T E Q / L) \text { using estimator }\end{array}$ & 0.015 & 0.01 & 0.01 & 0.01 & 0.215 & $\mathrm{BD}$ & 0.069 & 0.035 & 0.127 & NA & NA \\
\hline $\begin{array}{l}\text { method } \\
\text { Mean }(\mu \mathrm{g} T E Q / L) \text { of detected }\end{array}$ & NA & NA & NA & NA & NA & $\mathrm{BD}$ & 0.129 & NA & 0.108 & NA & NA \\
\hline values & 1 & 0 & 0.091 & 0 & 4.01 & $\mathrm{BD}$ & 0.233 & 0.035 & 0.159 & NA & NA \\
\hline Maximum ( $\mu \mathrm{g}$ TEQ/L) & 85 & 17 & 17 & 17 & 85 & $\mathrm{BD}$ & 1.676 & 0.049 & 0.851 & NA & NA \\
\hline Oldest sampling date & 2-6-1990 & $1-9-2004$ & $2-6-1990$ & $1-9-2004$ & $\begin{array}{l}11-14- \\
2005\end{array}$ & $\begin{array}{l}4-5- \\
2010\end{array}$ & $\begin{array}{l}11-11- \\
2005\end{array}$ & $\begin{array}{l}9-13- \\
2007\end{array}$ & $10-18-2005$ & NA & NA \\
\hline Most recent sampling date & $5-4-2017$ & $9-30-2016$ & $5-4-2017$ & $\begin{array}{l}9-30- \\
2016 \\
\end{array}$ & $\begin{array}{l}11-21- \\
2014\end{array}$ & $\begin{array}{l}4-5- \\
2010\end{array}$ & $\begin{array}{l}12-31- \\
2015\end{array}$ & $\begin{array}{l}3-18- \\
2009\end{array}$ & $8-26-2008$ & $\mathrm{NA}$ & NA \\
\hline \multicolumn{12}{|l|}{ Dissolved cPAHs } \\
\hline Number of results & 12 & 12 & 12 & 12 & 0 & 0 & 0 & 0 & 0 & 0 & 0 \\
\hline Percent of results below detection & 100 & 100 & 100 & 100 & NA & NA & NA & NA & NA & NA & NA \\
\hline Summary statistics estimator method & NA & NA & NA & NA & NA & NA & NA & NA & NA & NA & NA \\
\hline Minimum $(\mu \mathrm{g} T E Q / L)$ & $\mathrm{BD}$ & $\mathrm{BD}$ & $\mathrm{BD}$ & $\mathrm{BD}$ & NA & NA & NA & NA & NA & NA & NA \\
\hline $\begin{array}{l}\text { Minimum }(\mu \mathrm{g} \text { TEQ/L) of detected } \\
\text { values }\end{array}$ & $\mathrm{BD}$ & $\mathrm{BD}$ & $\mathrm{BD}$ & $\mathrm{BD}$ & NA & NA & NA & NA & NA & NA & NA \\
\hline $\begin{array}{l}\text { Median }(\mu \mathrm{g} \mathrm{TEQ} / \mathrm{L}) \text { using estimator } \\
\text { method }\end{array}$ & $\mathrm{BD}$ & $\mathrm{BD}$ & $\mathrm{BD}$ & $\mathrm{BD}$ & NA & NA & NA & NA & NA & NA & NA \\
\hline Median $(\mu \mathrm{g}$ TEQ/L) of detected & $\mathrm{BD}$ & $\mathrm{BD}$ & $\mathrm{BD}$ & $\mathrm{BD}$ & NA & NA & NA & NA & NA & NA & NA \\
\hline
\end{tabular}




\begin{tabular}{|c|c|c|c|c|c|c|c|c|c|c|c|}
\hline \multirow{3}{*}{ Parameter } & \multirow{2}{*}{\multicolumn{2}{|c|}{ Entire watershed }} & \multicolumn{4}{|c|}{ Duwamish subwatershed } & \multirow{2}{*}{\multicolumn{2}{|c|}{$\begin{array}{l}\text { Lower Green } \\
\text { subwatershed }\end{array}$}} & \multirow{3}{*}{$\begin{array}{c}\begin{array}{c}\text { Soos } \\
\text { subwatershed }\end{array} \\
\text { All }\end{array}$} & \multirow{2}{*}{\multicolumn{2}{|c|}{$\begin{array}{l}\text { Upper Green } \\
\text { subwatershed }\end{array}$}} \\
\hline & & & \multicolumn{2}{|c|}{ Lower Duwamish } & \multicolumn{2}{|c|}{ Upper Duwamish } & & & & & \\
\hline & All & $<1,000 \mathrm{ft}$ & All & $<1,000 \mathrm{ft}$ & All & $\begin{array}{c}<1,000 \\
\mathrm{ft}\end{array}$ & All & $<1,000 \mathrm{ft}$ & & All & $<1,000 \mathrm{ft}$ \\
\hline values & & & & & & & & & & & \\
\hline $\begin{array}{l}\text { Mean }(\mu \mathrm{g} \text { TEQ/L) using estimator } \\
\text { method }\end{array}$ & $\mathrm{BD}$ & $\mathrm{BD}$ & $\mathrm{BD}$ & $\mathrm{BD}$ & NA & NA & NA & NA & NA & NA & NA \\
\hline values & $\mathrm{BD}$ & $\mathrm{BD}$ & $\mathrm{BD}$ & $\mathrm{BD}$ & NA & NA & NA & NA & NA & NA & NA \\
\hline Maximum $(\mu \mathrm{g}$ TEQ/L) & $\mathrm{BD}$ & $\mathrm{BD}$ & $\mathrm{BD}$ & $\mathrm{BD}$ & NA & NA & NA & NA & NA & NA & NA \\
\hline Oldest sampling date & $\begin{array}{l}12-13- \\
2005\end{array}$ & $\begin{array}{l}12-13- \\
2005\end{array}$ & $\begin{array}{l}12-13- \\
2005\end{array}$ & 2005 & NA & NA & NA & NA & NA & NA & NA \\
\hline Most recent sampling date & $3-21-2006$ & $3-21-2006$ & $3-21-2006$ & $\begin{array}{l}3-21- \\
2006\end{array}$ & NA & NA & NA & NA & NA & NA & NA \\
\hline
\end{tabular}




\section{Polychlorinated Biphenyls}

Data for PCBs in groundwater were available in the lower two subwatersheds-Duwamish and Lower Green. Three unique parameters were reported: the sum of PCB Aroclors (PCB Aroclors), the sum of the 209 PCB congeners (PCB Congeners), and the sum of the 209 PCB congeners as TEQ (PCBs TEQ; fig. 5). There were 1,572 PCB Aroclor results; 1,179 (75 percent) of those were below detection (table 4). Detected values ranged from 0.0019 to $10 \mu \mathrm{g} / \mathrm{L}$. Most (98 percent) of the PCB Aroclor data were in the Duwamish subwatershed and of those, nearly all (99 percent) were in the Lower Duwamish subwatershed which includes the LDW. All the PCB Aroclor results in the Upper Duwamish subwatershed and in the Lower Green subwatershed were below detection. There were no Aroclor results in the Soos and Upper Green subwatersheds.

There were 54 results for PCB congeners in groundwater, reported as a summed concentration in picograms per liter (pg/L) and as a pg TEQ/L concentration (table 4). All results were from the Lower Duwamish subwatershed. The congener method has lower detection limits than the Aroclor method. Twenty-eight percent of the concentration results and 35 percent of the TEQ results were censored (most often because results were below detection). Detected concentrations ranged from 15.2 to 994,000 $\mathrm{pg} / \mathrm{L}$ or 0.0127 to $1.4 \mathrm{pg} \mathrm{TEQ/L} \mathrm{(table} \mathrm{4).} \mathrm{Thirty} \mathrm{of} \mathrm{the} 54$ results were from wells within 1,000 feet of the Green-Duwamish River. Detected concentrations in these samples ranged from 29.5 to $197,000 \mathrm{pg} / \mathrm{L}$ or 0.0107 to $1.4 \mathrm{pg} \mathrm{TEQ/L} \mathrm{(table} \mathrm{4).} \mathrm{Median} \mathrm{PCB} \mathrm{concentrations,} \mathrm{using} \mathrm{the} \mathrm{estimator} \mathrm{method} \mathrm{to} \mathrm{account}$ for censored data, were $167 \mathrm{pg} / \mathrm{L}$ for all data and $546 \mathrm{pg} / \mathrm{L}$ using only wells within 1,000 feet of the river. 


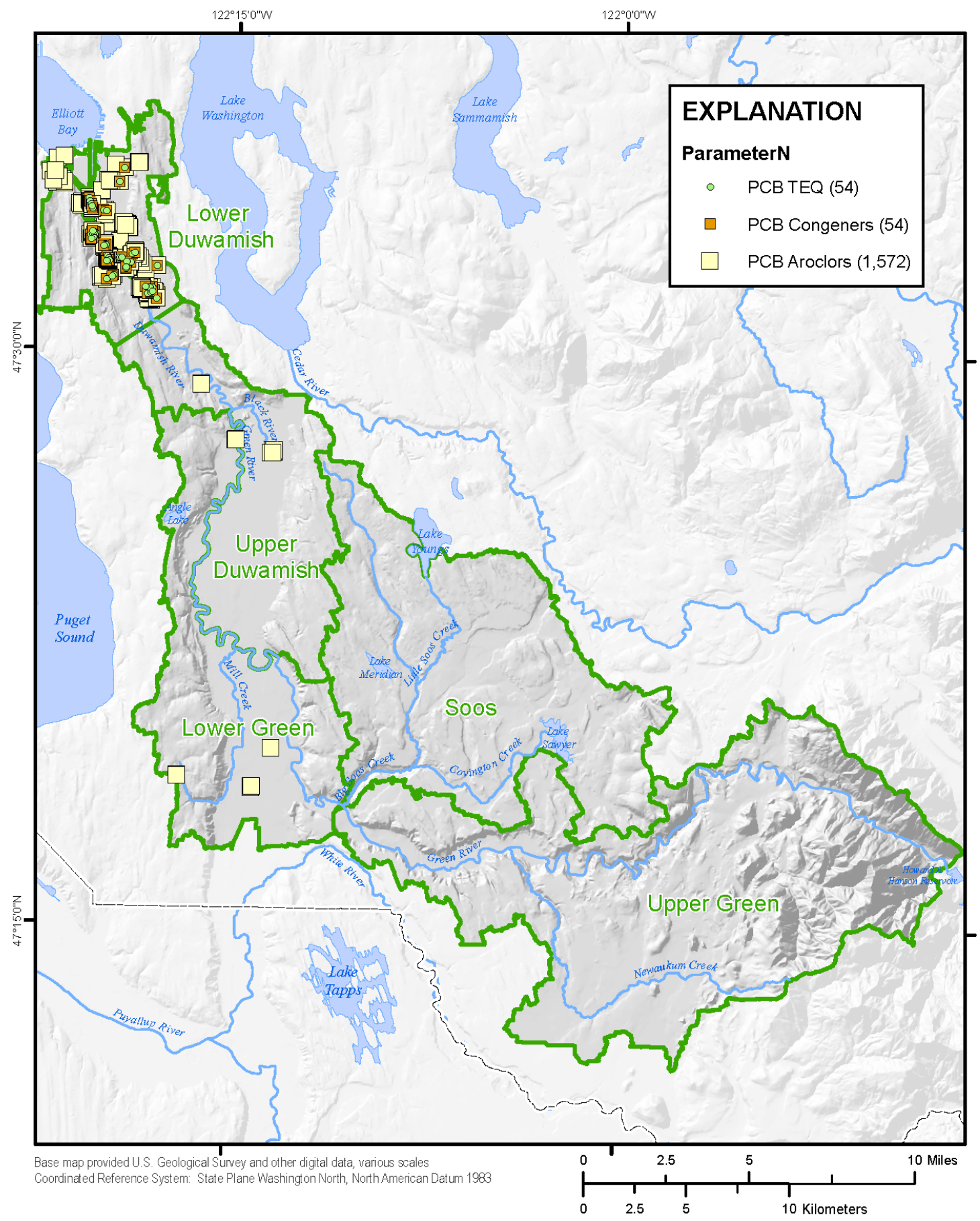

Figure 5. Map showing distribution of groundwater sites with polychlorinated biphenyl (PCB) data, GreenDuwamish watershed, Washington. 
Table 4. Summary of data for polychlorinated biphenyls (PCBs) in groundwater in the Green-Duwamish watershed, Washington.

[All: All results. $<\mathbf{1 , 0 0 0}$ ft: Results from wells within 1,000 feet of the Green-Duwamish River. Abbreviations: $\mu \mathrm{g} / \mathrm{L}$, micrograms per liter; $\mathrm{pg} / \mathrm{L}$, picograms per liter; MLE, maximum likelihood estimation; KM, Kaplan-Meier; NA, not applicable; BD, below detection.]

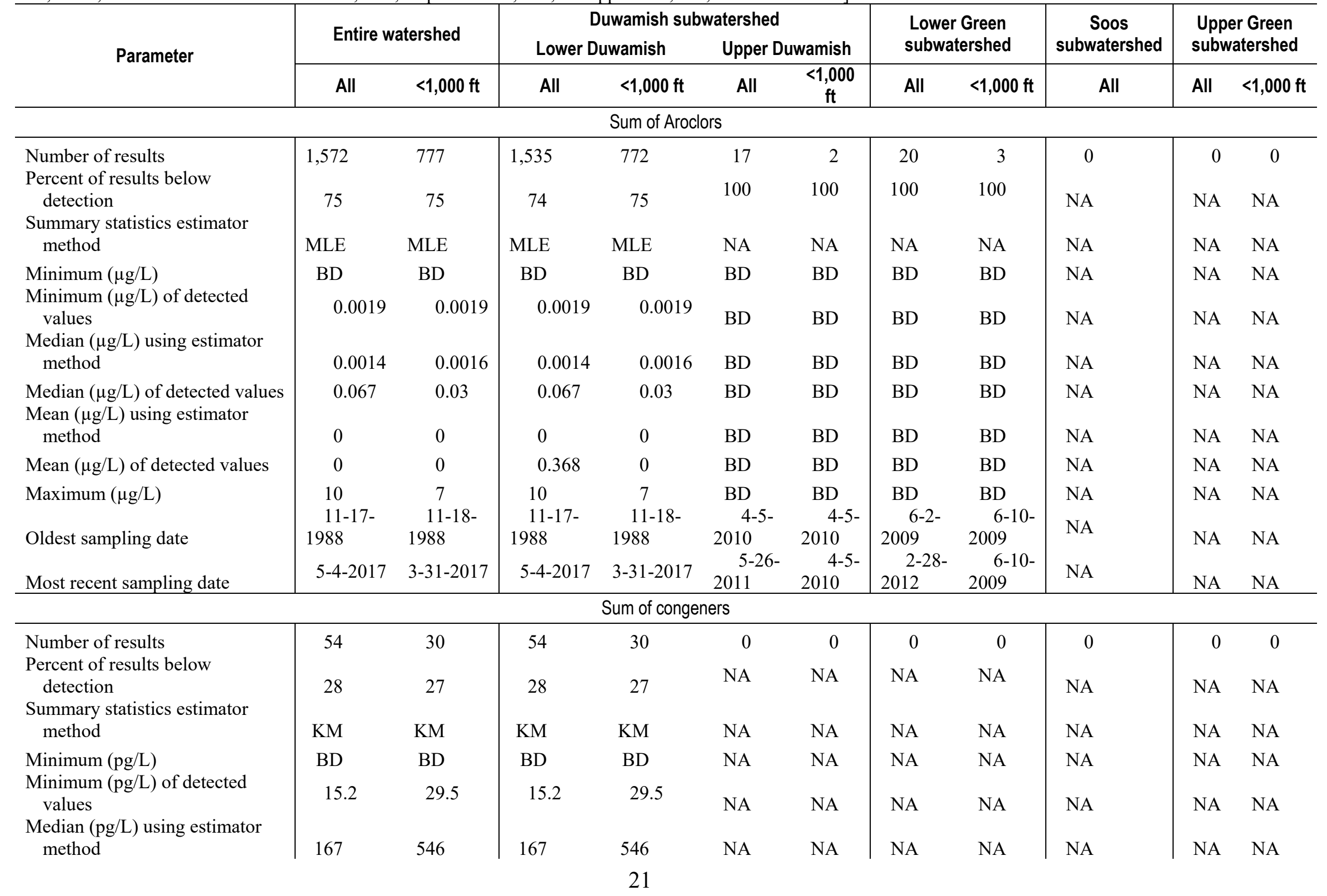




\begin{tabular}{|c|c|c|c|c|c|c|c|c|c|c|c|}
\hline \multirow{3}{*}{ Parameter } & \multirow{2}{*}{\multicolumn{2}{|c|}{ Entire watershed }} & \multicolumn{4}{|c|}{ Duwamish subwatershed } & \multirow{2}{*}{\multicolumn{2}{|c|}{$\begin{array}{l}\text { Lower Green } \\
\text { subwatershed }\end{array}$}} & \multirow{3}{*}{$\begin{array}{c}\begin{array}{c}\text { Soos } \\
\text { subwatershed }\end{array} \\
\text { All }\end{array}$} & \multirow{2}{*}{\multicolumn{2}{|c|}{$\begin{array}{l}\text { Upper Green } \\
\text { subwatershed }\end{array}$}} \\
\hline & & & \multicolumn{2}{|c|}{ Lower Duwamish } & \multicolumn{2}{|c|}{ Upper Duwamish } & & & & & \\
\hline & All & $<1,000 \mathrm{ft}$ & All & $<1,000 \mathrm{ft}$ & All & $\begin{array}{c}<1,000 \\
\mathrm{ft}\end{array}$ & All & $<1,000 \mathrm{ft}$ & & All & $<1,000 \mathrm{ft}$ \\
\hline Median (pg/L) of detected values & 1,115 & 824.00 & 1115 & 824.00 & NA & NA & NA & NA & NA & NA & NA \\
\hline method & 26,376 & 11,874 & 26,376 & 11,874 & NA & NA & NA & NA & NA & NA & NA \\
\hline Mean (pg/L) of detected values & 36,236 & 15,667 & 36,236 & 15,667 & NA & NA & NA & NA & NA & NA & NA \\
\hline Maximum (pg/L) & 994,000 & 197,000 & 994,000 & 197,000 & NA & NA & NA & NA & NA & NA & NA \\
\hline Oldest sampling date & $9-30-2013$ & $9-30-2013$ & $9-30-2013$ & $9-30-2013$ & NA & NA & NA & NA & NA & NA & NA \\
\hline Most recent sampling date & $4-6-2017$ & $3-31-2017$ & $4-6-2017$ & $3-31-2017$ & NA & NA & NA & NA & NA & NA & NA \\
\hline \multicolumn{12}{|c|}{ Sum of congeners reported in toxic equivalents (TEQs) } \\
\hline Number of results & 54 & 30 & 54 & 30 & 0 & 0 & 0 & 0 & 0 & 0 & 0 \\
\hline $\begin{array}{l}\text { Percent of results below } \\
\text { detection }\end{array}$ & 35 & 37 & 35 & 37 & NA & NA & NA & NA & NA & NA & NA \\
\hline $\begin{array}{l}\text { Summary statistics estimator } \\
\text { method }\end{array}$ & $\mathrm{KM}$ & $\mathrm{KM}$ & KM & KM & NA & NA & NA & NA & NA & NA & NA \\
\hline Minimum (pg TEQ/L) & $\mathrm{BD}$ & $\mathrm{BD}$ & $\mathrm{BD}$ & $\mathrm{BD}$ & NA & NA & NA & NA & NA & NA & NA \\
\hline $\begin{array}{l}\text { Minimum (pg TEQ/L) of } \\
\text { detected values }\end{array}$ & 0.0127 & 0.0107 & 0.0127 & 0.0107 & NA & NA & NA & NA & NA & NA & NA \\
\hline $\begin{array}{l}\text { Median (pg TEQ/L) using } \\
\text { estimator method }\end{array}$ & 0.0217 & 0.0201 & 0.0217 & 0.0201 & NA & NA & NA & NA & NA & NA & NA \\
\hline values & 0.0343 & 0.02 & 0.0343 & 0.02 & NA & NA & NA & NA & NA & NA & NA \\
\hline $\begin{array}{l}\text { Mean (pg TEQ/L) using } \\
\text { estimator method }\end{array}$ & 0 & 0 & 0 & 0 & NA & NA & NA & NA & NA & NA & NA \\
\hline $\begin{array}{l}\text { Mean }(\mathrm{pg} T E Q / L) \text { of detected } \\
\text { values }\end{array}$ & 0 & 0 & 0.153 & 0 & NA & NA & NA & NA & NA & NA & NA \\
\hline Maximum (pg TEQ/L) & 1 & 1 & 1 & 1 & NA & NA & NA & NA & NA & NA & NA \\
\hline Oldest sampling date & $9-30-2013$ & $9-30-2013$ & $9-30-2013$ & $9-30-2013$ & NA & NA & NA & NA & NA & NA & NA \\
\hline Most recent sampling date & $4-6-2017$ & $3-31-2017$ & $4-6-2017$ & $3-31-2017$ & NA & NA & NA & NA & NA & NA & NA \\
\hline
\end{tabular}




\section{Phthalates}

Bis(2-ethylhexyl)phthalate (BEHP) was used as an indicator compound for the phthalate group. Data for BEHP were available in the Duwamish subwatershed (fig. 6). There were 2,456 results compiled, of which 85 percent were below detection (table 5). Most (76 percent) of the phthalate data were in the Lower Duwamish. Detected concentrations ranged from 0.0538 to $410 \mu \mathrm{g} / \mathrm{L}$, and the maximum concentration reported from wells within 1,000 ft of the Green/Duwamish River was 89 $\mu \mathrm{g} / \mathrm{L}$. The median, mean, and maximum BEHP concentrations were higher in the Lower Duwamish than in the Upper Duwamish (table 5). 


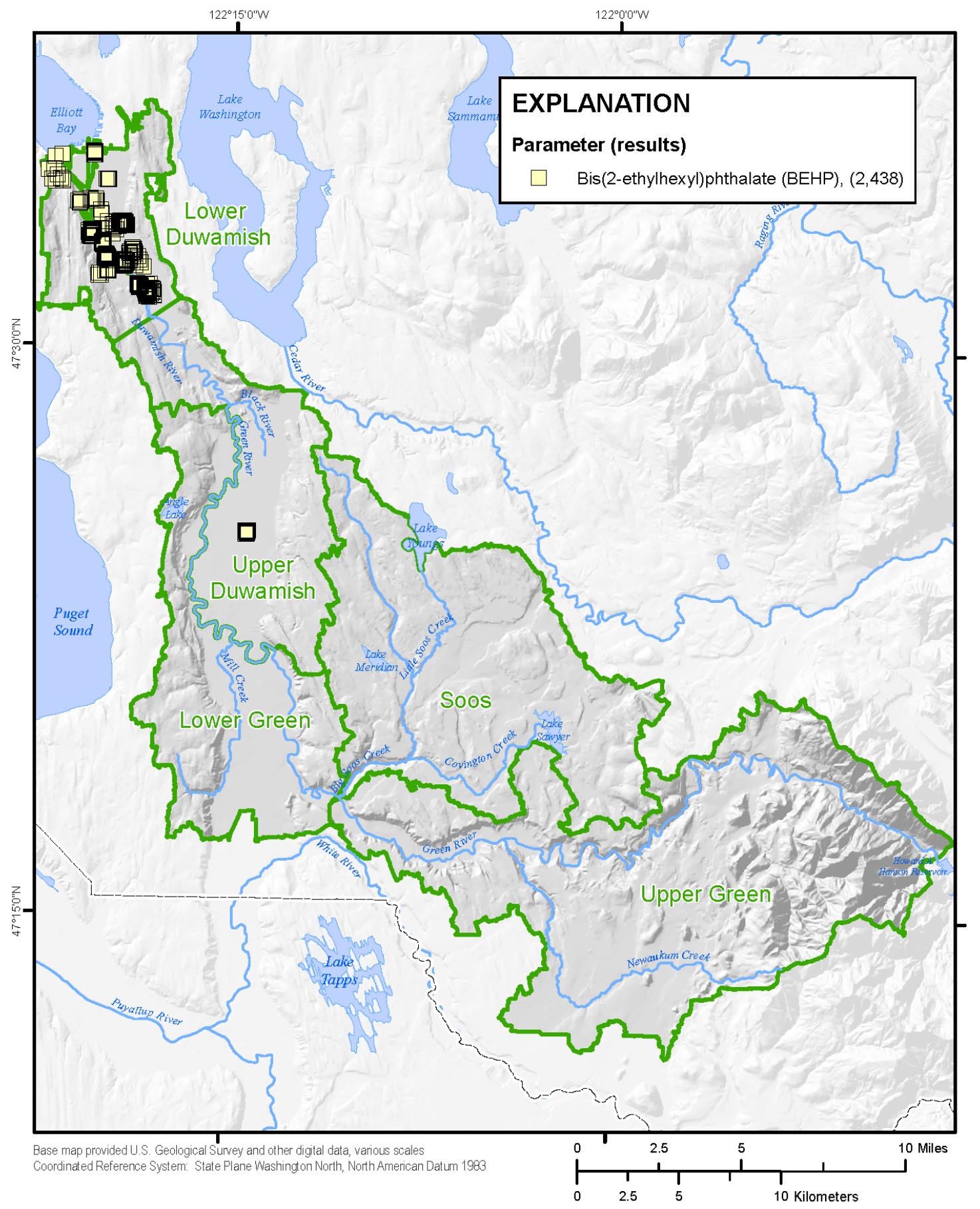

Figure 6. Map showing distribution of groundwater sites with phthalate data, Green-Duwamish watershed, Washington. 
Table 5. Summary of data for phthalates, represented by the indicator compound bis(2-ethylhexyl)phthalate, in groundwater in the GreenDuwamish watershed, Washington.

[All: All results. $<\mathbf{1 , 0 0 0 ~ f t : ~ R e s u l t s ~ f r o m ~ w e l l s ~ w i t h i n ~ 1 , 0 0 0 ~ f e e t ~ o f ~ t h e ~ G r e e n - D u w a m i s h ~ R i v e r . ~ A b b r e v i a t i o n s : ~ B D , ~ b e l o w ~ d e t e c t i o n ; ~ N A , ~ n o t ~ a p p l i c a b l e ; ~} \mu \mathrm{g} / \mathrm{L}$, micrograms per liter;]

\begin{tabular}{|c|c|c|c|c|c|c|c|c|c|c|c|}
\hline \multirow[t]{2}{*}{ Parameter } & \multicolumn{2}{|c|}{ Entire watershed } & \multicolumn{4}{|c|}{ Duwamish subwatershed } & \multicolumn{2}{|c|}{$\begin{array}{l}\text { Lower Green } \\
\text { subwatershed }\end{array}$} & \multirow{2}{*}{$\begin{array}{c}\begin{array}{c}\text { Soos } \\
\text { subwatersh } \\
\text { ed }\end{array} \\
\text { All }\end{array}$} & \multicolumn{2}{|c|}{$\begin{array}{l}\text { Upper Green } \\
\text { subwatershed }\end{array}$} \\
\hline & All & $<1,000 \mathrm{ft}$ & All & $<1,000 \mathrm{ft}$ & All & $<1,000 \mathrm{ft}$ & All & $<1,000 \mathrm{ft}$ & & All & $<1,000 \mathrm{ft}$ \\
\hline Number of results & 2,456 & 896 & 1,849 & 896 & 589 & 0 & 0 & 0 & 0 & 0 & 0 \\
\hline $\begin{array}{l}\text { Percent of results below } \\
\text { detection }\end{array}$ & 85 & 88 & 83 & 88 & 91 & NA & NA & NA & NA & NA & NA \\
\hline method & NA & NA & NA & NA & NA & NA & NA & NA & NA & NA & NA \\
\hline Minimum $(\mu \mathrm{g} / \mathrm{L})$ & $\mathrm{BD}$ & $\mathrm{BD}$ & $\mathrm{BD}$ & $\mathrm{BD}$ & $\mathrm{BD}$ & NA & NA & NA & NA & NA & NA \\
\hline $\begin{array}{l}\text { Minimum }(\mu \mathrm{g} / \mathrm{L}) \text { of detected } \\
\text { values }\end{array}$ & 0.0538 & 0.0538 & 0.0538 & 0.0538 & 0.13 & NA & NA & NA & NA & NA & NA \\
\hline $\begin{array}{l}\text { Median }(\mu \mathrm{g} / \mathrm{L}) \text { using estimator } \\
\text { method }\end{array}$ & NA & NA & NA & NA & NA & NA & NA & NA & NA & NA & NA \\
\hline values & 0.7 & 1.00 & 0.81 & 1.00 & 0.3 & NA & NA & NA & NA & NA & NA \\
\hline $\begin{array}{l}\text { Mean }(\mu \mathrm{g} / \mathrm{L}) \text { using estimator } \\
\text { method }\end{array}$ & NA & NA & NA & NA & NA & NA & NA & NA & NA & NA & NA \\
\hline Mean $(\mu \mathrm{g} / \mathrm{L})$ of detected values & 5 & 3 & 5.34 & 3 & 1.28 & NA & NA & NA & NA & NA & NA \\
\hline Maximum $(\mu \mathrm{g} / \mathrm{L})$ & 410 & 89 & 410 & 89 & 9.9 & NA & NA & NA & NA & NA & NA \\
\hline Oldest sampling date & $2-6-1990$ & $10-13-2004$ & 2-6-1990 & 10-13-2004 & $1-16-2006$ & NA & NA & NA & NA & NA & NA \\
\hline Most recent sampling date & $5-4-2017$ & $10-1-2016$ & $5-4-2017$ & $10-1-2016$ & $5-2-2017$ & NA & NA & NA & NA & NA & NA \\
\hline
\end{tabular}




\section{Zinc}

Zinc data were available in all four subwatersheds and included two parameters: zinc in unfiltered samples(zinc) and zinc in filtered samples (dissolved zinc; fig. 7). There were 3,162 zinc results in unfiltered samples, and 43 percent of those were below detection (table 6). All unfiltered zinc data were in the Duwamish subwatershed, with the majority (99 percent) of those within the Lower Duwamish. Detected concentrations ranged from 0.2 to $2,440 \mu \mathrm{g} / \mathrm{L}$. Like many of the other parameters, mean and maximum zinc concentrations were higher in the Lower Duwamish than in the Upper Duwamish (table 6).

There were 1,314 results for dissolved zinc, and 57 percent were below detection (table 6). Detected concentrations ranged from 0.45 to $930 \mu \mathrm{g} / \mathrm{L}$. Most (96 percent) of the dissolved zinc results were in the Lower Duwamish subwatershed. However, there were results for dissolved zinc in all subwatersheds, though the most recent samples collected in the Lower Green and Upper Green subwatersheds were in 1988. The maximum dissolved zinc concentration $(930 \mu \mathrm{g} / \mathrm{L})$ was in the Lower Duwamish subwatershed. The next highest concentration was in the Soos subwatershed $(370 \mu \mathrm{g} / \mathrm{L}$; table 6). Minimum, median, and mean dissolved zinc concentrations in the upper subwatersheds were similar to or higher than concentrations in the Lower Duwamish. 


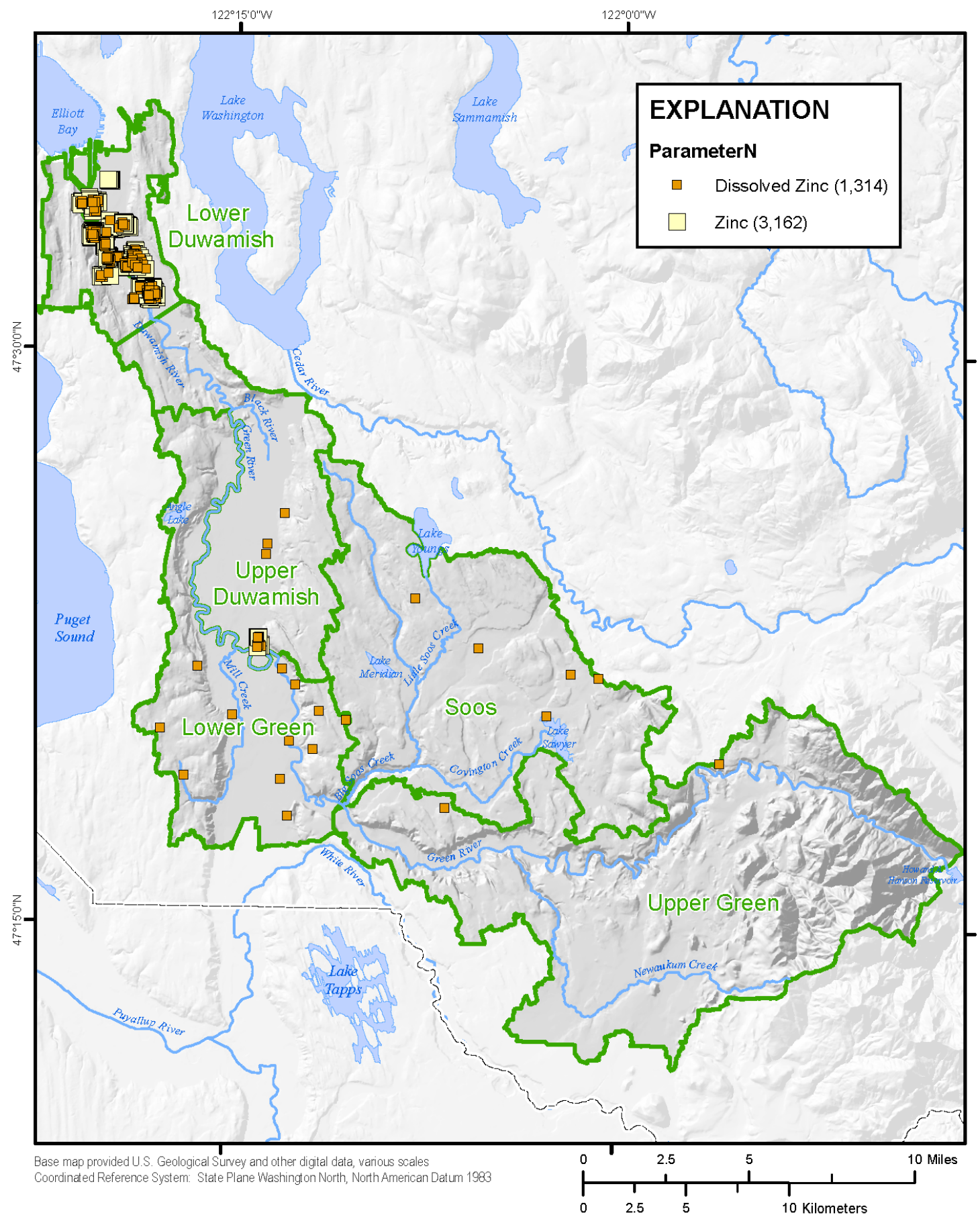

Figure 7. Map showing distribution of groundwater sites with zinc data, Green-Duwamish Watershed, Washington. 
Table 6. Summary of data for zinc in groundwater samples in the Green-Duwamish watershed, Washington.

[All: All results. $<\mathbf{1 , 0 0 0}$ ft: Results from wells within 1000 feet of the Green-Duwamish River. Abbreviations: $\mu \mathrm{g} / \mathrm{L}$, micrograms per liter; KM, Kaplan-Meier;

ROS, Regression on Order Statistics; NA, not applicable; BD, below detection.]

\begin{tabular}{|c|c|c|c|c|c|c|c|c|c|c|c|}
\hline \multirow{3}{*}{ Parameter } & \multirow{2}{*}{\multicolumn{2}{|c|}{ Entire watershed }} & \multicolumn{4}{|c|}{ Duwamish subwatershed } & \multirow{2}{*}{\multicolumn{2}{|c|}{$\begin{array}{l}\text { Lower Green } \\
\text { subwatershed }\end{array}$}} & \multirow{3}{*}{$\begin{array}{c}\begin{array}{c}\text { Soos } \\
\text { subwatershed }\end{array} \\
\text { All }\end{array}$} & \multirow{2}{*}{\multicolumn{2}{|c|}{$\begin{array}{l}\text { Upper Green } \\
\text { subwatershed }\end{array}$}} \\
\hline & & & \multicolumn{2}{|c|}{ Lower Duwamish } & \multicolumn{2}{|c|}{ Upper Duwamish } & & & & & \\
\hline & All & $<1,000 \mathrm{ft}$ & All & $<1,000 \mathrm{ft}$ & All & $<1,000 \mathrm{ft}$ & All & $<1,000 \mathrm{ft}$ & & All & $<1,000 \mathrm{ft}$ \\
\hline \multicolumn{12}{|c|}{ Unfiltered zinc } \\
\hline Number of results & 3,162 & 1,920 & 3,137 & 1,910 & 25 & 10 & 0 & 0 & 0 & 0 & 0 \\
\hline $\begin{array}{l}\text { Percent of results below } \\
\text { detection }\end{array}$ & 43 & 49 & 42 & 49 & 76 & 100 & NA & NA & NA & NA & NA \\
\hline $\begin{array}{l}\text { Summary statistics estimator } \\
\text { method }\end{array}$ & KM & KM & KM & KM & ROS & NA & NA & NA & NA & NA & NA \\
\hline Minimum $(\mu \mathrm{g} / \mathrm{L})$ & $\mathrm{BD}$ & $\mathrm{BD}$ & $\mathrm{BD}$ & $\mathrm{BD}$ & $\mathrm{BD}$ & $\mathrm{BD}$ & NA & NA & NA & NA & NA \\
\hline $\begin{array}{l}\text { Minimum }(\mu \mathrm{g} / \mathrm{L}) \text { of detected } \\
\text { values }\end{array}$ & 0.2 & 0.5 & 0.2 & 0.5 & 4 & $\mathrm{BD}$ & NA & NA & NA & NA & NA \\
\hline $\begin{array}{l}\text { Median }(\mu \mathrm{g} / \mathrm{L}) \text { using } \\
\text { estimator method } \\
\text { Median }(\mu \mathrm{g} / \mathrm{L}) \text { of detected }\end{array}$ & 3.5 & 3.86 & 3.76 & 3.9 & 5 & $\mathrm{BD}$ & NA & NA & NA & NA & NA \\
\hline $\begin{array}{l}\text { values } \\
\text { Mean }(\mu \mathrm{g} / \mathrm{L}) \text { using estimator }\end{array}$ & 7.2 & 9.40 & 7.1 & 9.40 & 9.5 & $\mathrm{BD}$ & NA & NA & NA & NA & NA \\
\hline & 24 & 26 & 19 & 26 & 6.86 & $\mathrm{BD}$ & NA & NA & NA & NA & NA \\
\hline values & 39 & 48 & 39.1 & 48 & 9.2 & $\mathrm{BD}$ & NA & NA & NA & NA & NA \\
\hline Maximum $(\mu \mathrm{g} / \mathrm{L})$ & 2,440 & 2,440 & 2,440 & 2,440 & 14 & $\mathrm{BD}$ & NA & NA & NA & NA & NA \\
\hline Oldest sampling date & $\begin{array}{l}11-17- \\
1988\end{array}$ & & $11-17-1988$ & $\begin{array}{l}11-17- \\
1988\end{array}$ & $5-30-2006$ & $5-30-2006$ & NA & NA & NA & NA & NA \\
\hline Most recent sampling date & $\begin{array}{l}5-24- \\
2018\end{array}$ & 2-14-2018 & $5-24-2018$ & $\begin{array}{l}2-14- \\
2018\end{array}$ & $5-19-2009$ & $8-29-2006$ & NA & NA & NA & NA & NA \\
\hline \multicolumn{12}{|c|}{ Dissolved zinc } \\
\hline Number of results & 1,314 & 941 & 1,266 & 928 & 24 & 10 & 15 & 3 & 7 & 2 & 0 \\
\hline $\begin{array}{l}\text { Percent of results below } \\
\text { detection } \\
\text { Summary statistics estimator }\end{array}$ & 57 & 60 & 58 & 60 & 75 & 100 & 7 & 0 & 43 & 0 & NA \\
\hline method & MLE & MLE & MLE & MLE & ROS & NA & $\mathrm{KM}$ & NA & KM & NA & NA \\
\hline Minimum $(\mu \mathrm{g} / \mathrm{L})$ & BD & $\mathrm{BD}$ & BD & BD & BD & BD & $\mathrm{BD}$ & 7 & $\mathrm{BD}$ & 48 & NA \\
\hline $\begin{array}{l}\text { Minimum }(\mu \mathrm{g} / \mathrm{L}) \text { of detected } \\
\text { values }\end{array}$ & 0.45 & 0.45 & 0.45 & 0.45 & 4 & $\mathrm{BD}$ & 3 & 7 & 5 & 48 & NA \\
\hline Median $(\mu \mathrm{g} / \mathrm{L})$ using & 2.49 & 5 & 2.38 & 2.29 & 8.19 & $\mathrm{BD}$ & 20 & NA & 5 & NA & NA \\
\hline
\end{tabular}




\begin{tabular}{|c|c|c|c|c|c|c|c|c|c|c|c|}
\hline \multirow{3}{*}{ Parameter } & \multirow{2}{*}{\multicolumn{2}{|c|}{ Entire watershed }} & \multicolumn{4}{|c|}{ Duwamish subwatershed } & \multirow{2}{*}{\multicolumn{2}{|c|}{$\begin{array}{l}\text { Lower Green } \\
\text { subwatershed }\end{array}$}} & \multirow{3}{*}{$\begin{array}{c}\begin{array}{c}\text { Soos } \\
\text { subwatershed }\end{array} \\
\text { All }\end{array}$} & \multirow{2}{*}{\multicolumn{2}{|c|}{$\begin{array}{l}\text { Upper Green } \\
\text { subwatershed }\end{array}$}} \\
\hline & & & \multicolumn{2}{|c|}{ Lower Duwamish } & \multicolumn{2}{|c|}{ Upper Duwamish } & & & & & \\
\hline & All & $<1,000 \mathrm{ft}$ & All & $<1,000 \mathrm{ft}$ & All & $<1,000 \mathrm{ft}$ & All & $<1,000 \mathrm{ft}$ & & All & $<1,000 \mathrm{ft}$ \\
\hline \multicolumn{12}{|l|}{ estimator method } \\
\hline \multirow{3}{*}{$\begin{array}{l}\text { Median }(\mu \mathrm{g} / \mathrm{L}) \text { of detected } \\
\text { values } \\
\text { Mean }(\mu \mathrm{g} / \mathrm{L}) \text { using estimator } \\
\text { method } \\
\text { Mean }(\mu \mathrm{g} / \mathrm{L}) \text { of detected } \\
\text { values }\end{array}$} & 6 & 6.00 & 6 & 6.00 & 11 & $\mathrm{BD}$ & 21.5 & 14 & 33 & NA & NA \\
\hline & 11 & 65 & 10 & 9 & 17.2 & BD & 26.5 & NA & 65.1 & NA & NA \\
\hline & 31 & 28 & 29.9 & 29 & 39 & $\mathrm{BD}$ & 27.9 & NA & 110 & 54 & NA \\
\hline Maximum $(\mu \mathrm{g} / \mathrm{L})$ & 930 & 930 & 930 & 930 & 180 & $\mathrm{BD}$ & 65 & 50 & 370 & 60 & NA \\
\hline Oldest sampling date & $\begin{array}{l}11-16- \\
1970\end{array}$ & $\begin{array}{l}10-21- \\
1971\end{array}$ & $5-18-2005$ & $\begin{array}{l}9-29- \\
2006\end{array}$ & $\begin{array}{l}12-11- \\
1971\end{array}$ & $5-30-2006$ & $\begin{array}{l}11-16- \\
1970\end{array}$ & $\begin{array}{l}3-4- \\
1988\end{array}$ & $5-16-1972$ & $\begin{array}{l}5-16- \\
1972\end{array}$ & NA \\
\hline Most recent sampling date & $\begin{array}{l}11-29- \\
2016\end{array}$ & $10-1-2016$ & $10-1-2016$ & $\begin{array}{l}10-1- \\
2016\end{array}$ & $1-26-2009$ & $8-29-2006$ & $\begin{array}{l}3-17- \\
1988\end{array}$ & $\begin{array}{l}3-11- \\
1988\end{array}$ & 11-29-2016 & $3-8-1988$ & NA \\
\hline
\end{tabular}




\section{Other Sources of Groundwater Chemistry Data}

The data summarized in this report was compiled from three sources: the USGS NWIS database, the Ecology EIM database, and from a data compilation of Green-Duwamish water-quality data by Leidos. Other possible sources of data include:

1. EPA's STORET;

2. Local or small-scale clean-up sites;

3. Construction projects; and

4. Federal cleanup projects.

The EPA's STORET database was not queried because of the expected high level of duplication with USGS and Ecology databases. However, there likely are additional groundwater chemistry data in STORET that could be retrieved in a future effort.

An additional source of groundwater chemistry data is from monitoring of clean-up sites in the watershed. These include various industrial and commercial sites, such as gas stations with leaking underground storage tanks, that require cleanup of one or more toxic chemicals. The cleanup process is tracked by Ecology, and often there is environmental monitoring, for example, of groundwater. On March 4, 2019, Ecology provided the USGS with a list obtained from the Washington State Toxics Cleanup web reporting tool (Washington State Department of Ecology, 2019) of all clean-up sites with confirmed or suspected contamination in Water Resources Inventory Area 9, which includes the GreenDuwamish watershed. The USGS sorted the list to include only sites within the PLA-defined watershed boundary and with known or suspected groundwater contamination by the following chemical names or groups: Arsenic, Metals Priority Pollutants, Polycyclic Aromatic Hydrocarbons, and Polychlorinated biPhenyls. Copper and zinc likely were included in the Metals Priority Pollutants category. There was not a phthalate group. A further review of the individual parameters included in each category would determine whether copper and zinc are part of the Metals Priority Pollutants and might identify some data for individual phthalates.

The sorting resulted in 185 sites (fig. 8, grey circles) in the Green-Duwamish watershed with known or suspected groundwater contamination for one of the chemical groups (Arsenic, Metals Priority Pollutants, Polycyclic Aromatic Hydrocarbons, and Polychlorinated biPhenyls). The 185 sites were mapped as a new layer overlaying the map layers of sites from the Leidos+EIM+NWIS data pull. Many of the 185 sites appeared to be new locations that were not included in the Leidos+EIM+NWIS data pull (fig. 8, grey symbols are the 185 cleanup sites and open circles are the Leisos+EIM+NWIS sites). Sites within 1,000 feet of the Green/Duwamish River were further denoted (fig. 8, red triangles). The analysis identified:

1. Upper Green: Five sites, including one site with suspected PCBs and PAHs above cleanup levels and four sites with suspected or confirmed metals concentrations above cleanup levels. All five Upper Green sites were greater than 1,000 ft from the Green River.

2. Soos: Seven sites, including one site with suspected PCBs and PAHs above cleanup levels and six sites with suspected or confirmed metals concentrations above cleanup levels (one of which had confirmed PCBs above cleanup level).

3. Lower Green: Ten sites, including three within 1,000 ft of the Green River. Of the three sites within 1,000 $\mathrm{ft}$ of the Green River, all are awaiting clean-up for suspected metals and one for PAHs.

4. Upper Duwamish: 45 sites, including seven within 1,000 ft of the Green/Duwamish River. Six of the seven sites had confirmed or suspected metals concentrations above cleanup level (one also 
had confirmed elevated arsenic) and the seventh site had confirmed arsenic concentrations above the cleanup level.

5. Lower Duwamish: 118 sites, including 22 sites within 1,000 ft of the Duwamish River.

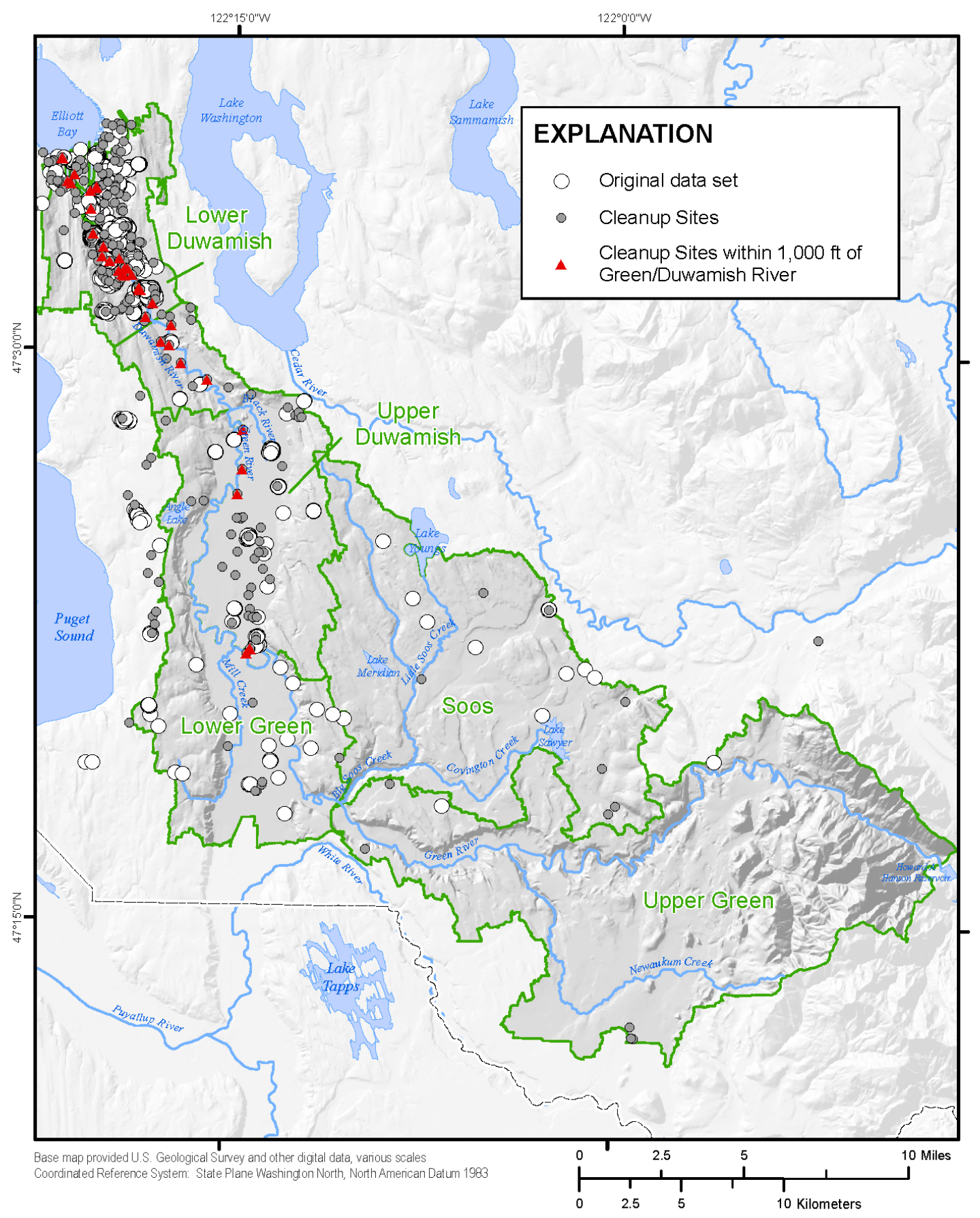

Figure 8. Map showing distribution of groundwater data from designated cleanup sites, Green-Duwamish watershed, Washington. 
Site information for a subset of the 185 sites, including reports and data, was queried in May 2019 from Cleanup Site Search (Washington Department of Ecology, 2019b; particularly the "View Electronic Documents" on the right tab), and Toxic Cleanup Program Web Reporting (Washington Department of Ecology, 2019c), which did not always have the most current information, but provided basic information about the site when no other documents were available. The availability of current information and data varied by site, and when data was available, it was usually in PDF form rather than a readily exportable form such as .csv or .xlsx file. This exercise identified cleanup sites with groundwater data for the parameters of concern that do not appear to be in one of the compiled databases (EIM, NWIS, Leidos compilation). Future work could include a compilation of relevant data from selected cleanup sites, for example, based on location and cleanup status.

Other potential data sources of groundwater chemistry data in the Green-Duwamish watershed include the Washington State Department of Transportation, the Port of Seattle, and the Seattle Department of Transportation. There may be groundwater monitoring data incorporated into the environmental assessments of construction projects involving the removal and off-site disposal of soils.

Another potential source of groundwater data for the chemicals of interest is from other federal cleanup projects - for example, cleanup sites on Puget Sound Naval facilities. While the data would be outside of the Green-Duwamish watershed, some values (for example, post-remediation monitoring), may be representative of groundwater conditions in the Puget lowlands glacial till found in the GreenDuwamish basin. Like the Toxics Cleanup sites, data from these city, state, and federal projects typically are not in a publicly-available database and would require acquiring the reports of the environmental assessments and manually exporting relevant groundwater data.

\section{Data Gaps and Needs for Future Study}

The data compilation of groundwater chemistry data in the Green-Duwamish watershed identified 22,520 total results from 1,607 sites for the six parameter groups of interest: arsenic, copper, cPAHs, PCBs, phthalates, and zinc. All but 296 results were in the Duwamish subwatershed, and the majority of those were in the Lower Duwamish which contains the LDW. This suggests that representative groundwater concentrations may be able to be assigned in the PLA model's Hydrologic Response Units in the Duwamish subwatershed, though further analysis of the available data is recommended. For example, data could be examined for variables such as:

1. Sampling date: Samples collected more recently are assumed to be more representative of current groundwater conditions than older samples. Samples from pre-remediation should be removed and only post-remediation values retained. Also, the data are more likely to have been generated with modern collection and analysis methods.

2. Data quality: In this report, the result qualifier was reported (for example, "U" indicating a value was not detected), but no additional information on data quality was available. One important consideration is how blank contamination is accounted for, especially for low-level analysis. Many labs report environmental results, and it is the user's responsibility to blank-correct, either by doing a blank-subtraction or by censoring as non-detects, environmental results near the blank concentration.

3. Subsurface characteristics: The depth to groundwater, the depth from which the sample was collected, and the subsurface lithology where the well is located are critical to understanding relevance of well groundwater quality to groundwater that may discharge to the river.

4. Other water-quality parameters: Characteristics such as specific conductance, organic carbon, suspended solids concentration, and dissolved oxygen can influence occurrence and concentrations of chemical parameters. 
Limited groundwater chemistry data were available in the three upper subwatersheds. In the Upper Green, there was no data available for cPAHs, PCBs, or phthalates and one to two results for each of the three metals. Those results were from 1988 from sites further than 1,000 ft from the river. In the Soos subwatershed, there were no data available for PCBs and phthalates. The cPAH results were mostly below detection, and the most recent results were from 2008 . There were less than 10 results each for dissolved arsenic, dissolved copper, and dissolved zinc (and one result for total arsenic). In the Lower Green subwatershed, there were no data for phthalates and PCB congeners. There were 20 PCB Aroclor results, all of which were below detection. There were more than $100 \mathrm{cPAH}$ results, from as recently as 2015. There were approximately 15 results for each of the three dissolved metals from 1988, and 14 total arsenic results from 2009. The analysis suggests that additional groundwater chemistry data is needed in the three upper subwatersheds: Upper Green, Soos, and Lower Green.

To fill a known data gap of PCB data in groundwater discharging to the LDW, Leidos (2017) analyzed groundwater samples from 17 properties within the LDW basin that were currently undergoing environmental investigations or cleanups. At each property, when feasible, a transect of three wells were sampled, including a well upland of contamination, a well within the contamination, and a well near the river. The PCB concentration, as a sum of 209 congeners, in 7 of the upland wells, ranged from 15.2 to $50.6 \mathrm{pg} / \mathrm{L}$, and for modeling may be representative of background or ambient concentrations of PCBs in groundwater in the urban and industrial portions of the watershed, in the absence of other data. There are no PCB congener data in other portions of the watershed, including residential, agricultural and forested sub-basins. Therefore, it is unknown if the available upland data is representative of other portions of the watershed.

Future work could include querying additional potential data sources, such as EPA's STORET database, construction project environmental assessments by the Washington State Department of Transportation, the Port of Seattle, and the Seattle Department of Transportation, and relevant Puget Sound basin projects such as Navy remediation projects. One method for estimating the concentrations of groundwater discharging into surface water in the upper subwatersheds where there is little or no available data is to compile or sample surface water concentration data during periods of low flow. These streams are sustained by groundwater inflow during summer low-flow periods, and therefore, represent the collective groundwater inflow upstream from the sampling point. If many samples are collected along the length of the tributaries and main stem, differences in concentrations in a downstream direction result partly from gains from or losses to groundwater. For example, King County (2018) sampled surface water during three baseflow events from four major tributaries-one in each subwatershed. Samples were analyzed for total and dissolved arsenic, PAHs, and PCBs. Mean total arsenic concentrations in these baseflow surface water samples ranged from $0.647 \mathrm{ug} / \mathrm{L}$ in Newaukum Creek to $1.05 \mathrm{ug} / \mathrm{L}$ in Soos Creek, and mean PCB concentrations (as a sum of congeners) ranged from $177 \mathrm{pg} / \mathrm{L}$ in Newaukum Creek to $1,590 \mathrm{pg} / \mathrm{L}$ in Soos Creek. The results indicated there is a source of PCBs to the Soos Creek sampling location-during baseflow conditions, the highest single total PCB concentration was detected in Soos Creek $(4,680 \mathrm{pg} / \mathrm{L})$. Polycyclic aromatic hydrocarbons were not reported as cPAHs in TEQ. The results for individual compounds are available in the appendix tables, so a cPAH TEQ calculation could be done. The results indicate that there are detectable PAHs (particularly the high molecular-weight PAHs) at the Black River sampling location in the Upper Duwamish subwatershed. These baseflow surface water samples likely represent a composite of chemical contributions from groundwater, atmospheric deposition, and other contributions to surface water (such as partitioning from bed sediment and illicit discharges). 
This data compilation emphasized the importance of specifying the parameter of interest, including chemical speciation and sample handling. For example, results from unfiltered and filtered samples are unique and cannot be combined into a single data set. Further, the speciation of metals such as arsenic needs to be specified, as results from different species (for example, total, dissolved, inorganic, arsenic III) are not interchangeable.

Summary statistics were calculated on results from sites within 1,000 feet of the GreenDuwamish River on the presumption that these may be more representative of concentrations discharging to the river than sites further inland. However, this is just a hypothesis in the absence of knowledge of actual groundwater direction and flow. Modeling of groundwater levels, directions, and flow paths has not been done for the Green-Duwamish watershed, and is a critical data gap to estimating chemical load contributions from groundwater to the river.

\section{Summary}

This report summarizes available groundwater data for six parameters of interest: arsenic, copper, cPAHs, PCBs, phthalates, and zinc, compiled from three data sources: the USGS NWIS database, Ecology's EIM database, and a database of LDW chemistry data by Leidos. Summary statistics by parameter and subwatershed were reported. Data gaps and future study needs were described, including potential sources of additional data, the need for additional data in the three upper subwatersheds, and the need for an improved understanding of the groundwater directions and flows in relation to the Green-Duwamish River. This information will support Ecology's source control efforts by improving understanding of the role of groundwater in contributing loads of chemicals to the GreenDuwamish River.

\section{References Cited}

Fabritz, J., Massmann, J., and Booth, D., 1998, Development of a three-dimensional, numerical groundwater flow model for the Duwamish River basin: Seattle, University of Washington, Center for Urban Water Resources Management, 59 p.

Helsel, D.R., 2005, Nondetects and data analysis: Hoboken, N.J., John Wiley \& Sons, Inc., 250 p.

King County, 2018, Lower Duwamish waterway source control: Green River Watershed Surface Water Data Report, originally published 2014 and revised 2018, 100 p., accessed June 27, 2019 at https://www.kingcounty.gov/services/environment/wastewater/duwamish-waterway/preventingpollution/pollution-sources.aspx.

Lee, L., 2017, Package "NADA"-Nondetects and data analysis for environmental data (version 1.6-1): R Foundation for Statistical Computing, accessed June 27, 2019, at https://CRAN.Rproject.org/package $=$ NADA.

Leidos, 2017, Lower Duwamish Waterway-Groundwater sampling for PCB congeners and Aroclors: Data Report, Final, Prepared for the Washington Department of Ecology Toxic Cleanup Program, 256 p.

R Core Team, 2019, The R project for statistical computing: Vienna, Austria, R Foundation for Statistical Computing, accessed June 27, 2019, at https:/www.r-project.org/.

U.S. Environmental Protection Agency, 2014, Record of decision-Lower Duwamish Waterway Superfund site: U.S. Environmental Protection Agency, accessed June 25, 2019, at https://semspub.epa.gov/work/10/715975.pdf. 
Washington Department of Ecology, 2014, Focus on Green-Duwamish-A pollutant loading assessment for the Green-Duwamish Watershed: Washington Department of Ecology 14-10-053, 3 p., accessed June 26, 2019 at https://fortress.wa.gov/ecy/publications/SummaryPages/1410053.html.

Washington Department of Ecology, 2019a, Toxics cleanup program web report: Washington Department of Ecology, web.

Washington Department of Ecology, 2019b, Cleanup site search: Washington Department of Ecology, web.

Washington Department of Ecology, 2019c, Search for cleanup sites in Washington state: Washington Department of Ecology, web.

Welch, W.B., Johnson, K.H., Savoca, M.E., Lane, R.C., Fasser, E.T., Gendaszek, A.S., Marshall, C., Clothier, B.G., and Knoedler, E.N., 2015, Hydrogeologic framework, groundwater movement, and water budget in the Puyallup River Watershed and vicinity, Pierce and King Counties, Washington: U.S. Geological Survey Scientific Investigations Report 2015-5068, 54 p., 4 pls. [Also available at http://dx.doi.org/10.3133/sir20155068.] 

Publishing support provided by the U.S. Geological Survey Science Publishing Network, Tacoma Publishing Service Center

For more information concerning the research in this report, contact the Director, Washington Water Science Center

U.S. Geological Survey

934 Broadway, Suite 300

Tacoma, Washington 98402

https://www.usgs.gov/centers/wa-water 


\section{$\mathbb{N}$ \\ 密}

趈

产

을

呄

兽

蛋

ㄸ.

官.

몽

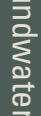

을

票

뮴

言

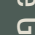

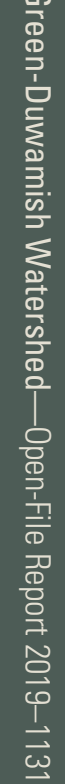

ISSN 2331-1258 (online) 